\title{
Knowledge Translation in Mental Health: A Scoping Review
}

\section{Échange de connaissances en santé mentale : analyse de la situation}

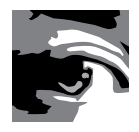 \\ ELLIOT M. GOLDNER, MHSC, MD \\ Professor, Centre for Applied Research in Mental Health $\&$ Addiction \\ Faculty of Health Sciences, Simon Fraser University \\ Vancouver, BC \\ VICTORIA JEFFRIES, MA \\ Student, Centre for Applied Research in Mental Health $\&$ Addiction \\ Faculty of Health Sciences, Simon Fraser University \\ Vancouver, BC \\ DAN BILSKER, PHD \\ Adjunct Professor, Centre for Applied Research in Mental Health $\&$ Addiction \\ Faculty of Health Sciences, Simon Fraser University \\ Vancouver, BC \\ EMILY JENKINS, RN, MPH \\ Student, School of Nursing, University of British Columbia \\ Vancouver, BC \\ MATTHEW MENEAR, MPH \\ Student, Department of Social and Preventive Medicine \\ Faculty of Medicine, University of Montreal \\ Montreal, QC \\ LISA PETERMANN, PHD \\ Director, Knowledge Exchange Centre \\ Mental Health Commission of Canada \\ Calgary, $A B$
}




\begin{abstract}
Intensified knowledge translation (KT) efforts are considered important in the field of mental health in order to accelerate the implementation of various developments in research, policy and practice. A scoping review of KT focused on the field of mental health was undertaken to help inform development of a Knowledge Exchange Centre being initiated by the Mental Health Commission of Canada. A systematic search of publications in English and French identified 187 publications that met inclusion criteria. Relevant literature was found across a number of disparate thematic research areas: implementation science, community-based and participatory action research, shared decision-making studies, mental health literacy research, network analysis and studies directly addressing KT. The available literature is concerned predominantly with KT efforts between a few specific stakeholder dyads. A paradigm shift has been emerging and has resulted in a progressively broader perspective, incorporating a wider range of participants and increased valuing of experiential knowledge.
\end{abstract}

\title{
Résumé
}

On considère que les efforts d'échange de connaissances (EC) dans le domaine de la santé mentale sont essentiels pour accélérer la mise en œuvre de diverses avancées dans la recherche, les politiques et la pratique. Une analyse de la situation des activités d'EC dans le domaine de la santé mentale a été effectuée pour éclairer la création d'un Centre d'échange des connaissances étant initié par la Commission de la santé mentale du Canada. Une recherche systématique des publications en anglais et en français a permis de répertorier 187 titres qui répondaient aux critères d'inclusion. La littérature pertinente était classée sous diverses thématiques de recherche : science de mise en œuvre, activités de recherches participatives axées sur la communauté, études sur la prise de décision partagée, recherche sur les compétences informationnelles en santé mentale, analyse des réseaux ou études qui traitent directement de l'EC. Cette littérature porte principalement sur les efforts d'EC qui mettent en jeu quelques paires d'intervenants particuliers. Un changement de paradigme présentement en cours a donné lieu à une vue d'ensemble de plus en plus vaste, en incorporant une plus grande gamme de participants et en valorisant de plus en plus les connaissances expérientielles.

\section{$\mathrm{T}$}

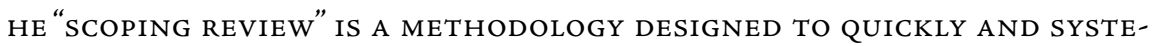
matically identify the breadth of literature in an area being researched. It has four major functions: (a) to map the current state of literature in an area of interest, (b) to determine the usefulness or feasibility of conducting a systematic review, (c) to summarize and disseminate research findings to an audience (e.g., policy makers, healthcare providers) and (d) to identify gaps or areas where further research is required (Arksey and O'Malley 2005; Levac et al. 2010). It may be contrasted with the systematic review, which is more restricted in focus and seeks to answer particular research questions from the available literature. 
This scoping review was undertaken to inform strategic planning for the development of a Knowledge Exchange Centre (KEC) - a component of the Mental Health Commission of Canada, a national organization created in 2007 through a federal endowment with a 10-year mandate to help improve mental health in Canada. The KEC, now being developed, will constitute one of the Commission's key initiatives and will seek to mobilize knowledge that will bring about action aimed to achieve meaningful and robust improvements to mental health in Canada. Development of the strategic plan for the KEC involved a review of the existing literature addressing knowledge translation (KT) focused on mental health issues.

The following definition of KT, advanced by the Canadian Institutes of Health Research (2010), has been widely adopted in the field of healthcare and was used in this study:

[A] dynamic and iterative process that includes synthesis, dissemination, exchange and ethically-sound application of knowledge to improve the health of Canadians, provide more effective health services and products and strengthen the health care system. This process takes place within a complex system of interactions between researchers and knowledge users.

Interest in $\mathrm{KT}$ is not confined to the field of healthcare and has permeated many disciplines, including education, marketing, engineering, economics, information technology, community development and various business enterprises. In regard to health services research and health policy, Canadian contributors have often been at the forefront of developments in KT research, theory and practice (e.g., Barwick et al. 2009; Dobbins et al. 2009; Driedger et al. 2010; Gagnon 2009; Goering et al. 2003; Grimshaw et al. 2001; Jacobson et al. 2003; Lavis et al. 2006; Légaré et al. 2010; Lomas 1993, 2000; McGrath et al. 2009; Mitton et al. 2007; Straus et al. 2009; Waddell et al. 2005). The potential value of KT in achieving improvements in healthcare delivery and health outcomes has generated substantial enthusiasm, and KT has become a prominent element within Canadian health research funding organizations (e.g., Lomas 2000; Graham and Tetroe 2009). Evidence for the effectiveness of KT is accumulating gradually, but remains limited (Mitton et al. 2007). In approaching this scoping review of mental health-related KT we sought to cast a wide net, examining a broad range of findings, ideas and developments, while also aiming to identify gaps, unanswered questions and challenges that might emerge in reviewing the published literature.

\section{Methods}

This scoping review followed five steps:

\section{Development of research questions}

Three questions guided selection of relevant sources: (a) What is the nature of research activity concerning KT focused on mental health? (b) What types of KT initiatives have been 
studied in relation to mental health? and (c) What is the nature of research activity concerning processes of KT among stakeholders relevant to mental health?

\section{Location of relevant publications}

A total of 22 terms were used to search 11 electronic databases of published scientific literature. Search terms included knowledge exchange, knowledge transfer, knowledge translation, knowledge linkage, knowledge mobilization, knowledge management, knowledge sharing, knowledge utilization, knowledge distribution, knowledge diffusion, knowledge realization, knowledge embodiment, knowledge sharing + transfer, implementation research, research + use, information sharing, knowledge economy, knowledge level, organizational behaviour, evidence-based practice, diffusion of innovation and action research. An initial literature search was undertaken in English and, subsequently, a search of the French literature using translations of these terms was carried out. This latter search took into account linguistic differences, such as single English terms being captured by multiple French terms (e.g., knowledge being referred to as connaissance or savoir).

The 11 electronic databases searched included PsycINFO, CINAHL, Consumer Health Complete, EconLit, Global Health, Health Source - Consumer and Nursing/Academic editions, MEDLINE, SocINDEX, PubMed and Érudit. This search procedure gathered approximately 10,000 abstracts. These abstracts were then searched for keywords related to mental health, including mental, psyc (an abbreviation appropriate for both psychology- and psychiatry-related papers), depression, schizophrenia and anxiety. This secondary search reduced the list of abstracts to 326 . These remaining abstracts were manually reviewed by members of the research team using the screening tool described in the next section. Papers were included if they addressed $\mathrm{KT}$ in the area of mental health and were published in peer-reviewed journals or dissertation abstracts in English or French. All papers were included regardless of publication date. We focused on the area of mental health broadly but excluded papers focused on low-income countries because of the likely differences in the structures and processes of exchange and communication of knowledge from those that exist in Canada.

Following these steps, 123 abstracts were identified initially as meeting our inclusion criteria. Preliminary analysis of the abstracts and discussion identified additional research areas as likely places to find relevant publications that did not appear in the initial round of searches. We also hand-searched reference lists of highly relevant papers and included those known to the authors, collecting an additional 64 abstracts. We chose to include dissertations with published literature rather than grey literature because of the substantial differences in audience for publicly available reports and unpublished dissertations, which can often be found only via university library memberships.

\section{Screening and selection of publications}

A screening tool was developed to determine relevance of papers to the exchange of knowledge related to mental health and code the type of data provided (e.g., empirical data, reviews, 
theoretical discussions). At the end of this process, 187 papers were deemed relevant, with $82 \%$ inter-rater agreement. Raters met to discuss disagreements in coding, and decisions about including or excluding papers were made jointly.

\section{Organizing publications by use of appropriate framework}

The papers were organized and coded as per the scoping review process proposed by Arksey and O'Malley (2005). Each of the 187 publications was categorized by country based on the location of the first author. We categorized the research approach as either quantitative, qualitative, discussion or other and included additional descriptors of research design based on design characteristics that were stated within the papers when these were included.

The publications were also categorized utilizing the knowledge exchange framework adopted by the Mental Health Commission of Canada (see Figure 1) in regard to the stakeholder groups that the KT addressed. The six stakeholder groups are as follows: (a) people with lived experience (PWLE) of mental health problems, which is a group that is broader than "patients," "clients" or "service-users," because the group is not limited to those who receive services but includes anyone who has experienced mental health problems directly; (b) family caregivers, i.e., family and friends of people with lived experience who may provide care and support; (c) policy makers; (d) researchers; (e) providers of mental health care; and (f) other stakeholders who interact with people suffering from mental health problems, e.g., employers, police or the general public. Publications were classified in regard to type of exchange, identifying KT to have occurred either within one group of stakeholders, i.e., within group; between two groups, i.e., dyad; or across three or more stakeholder groups, i.e., polyad. The stakeholder groups that were involved were identified.

\section{Elucidation of key themes}

This scoping review enabled identification of key themes emerging from within the existing literature on mental health-related KT. By illuminating these themes, we were able to examine key issues, areas of focus and potential gaps.

\section{Results}

A full listing of the 187 publications obtained through our systematic search procedures in tabular form, organized by publication date (from earliest to most recent year of publication) and classified into the categories described in this section is available online at: http://www. longwoods.com/content/22620.

\section{Year of publication}

Figure 2 shows our findings regarding the publication dates of the papers that were selected in this review. Most papers were published relatively recently; the majority appeared in the literature over the four-year period immediately preceding this study, i.e., from 2007 to 2010. 
FIGURE 1. The knowledge exchange framework adopted by the Mental Health Commission of Canada describes the exchange of knowledge across six stakeholder groups

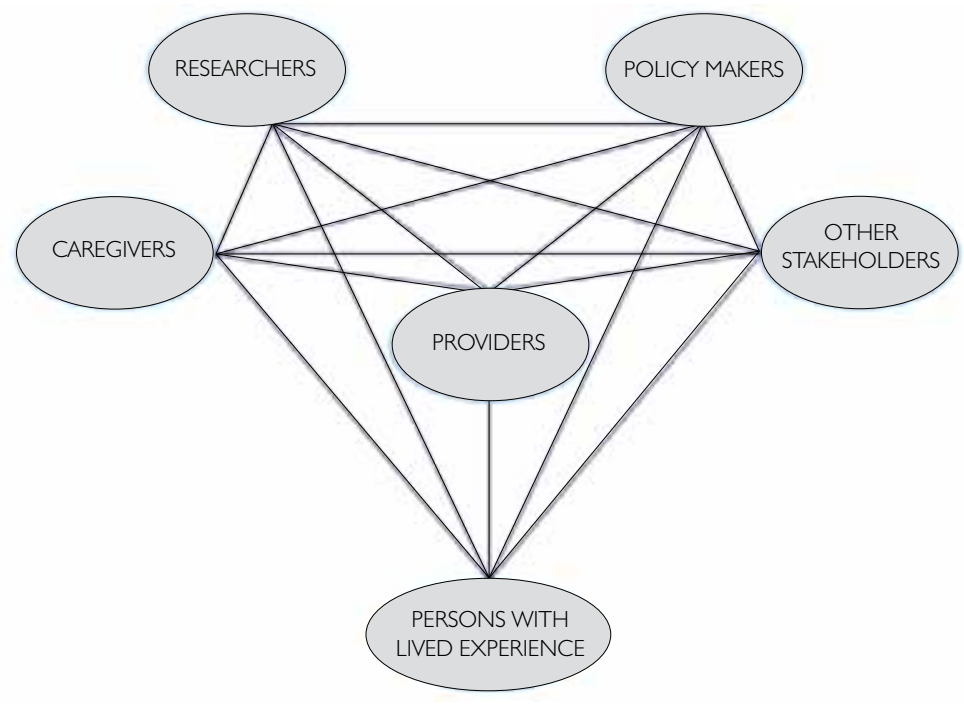

FIGURE 2. Year of publication of mental health-related KT publications obtained in the systematic search of the literature in five-year blocks, 1981-2010

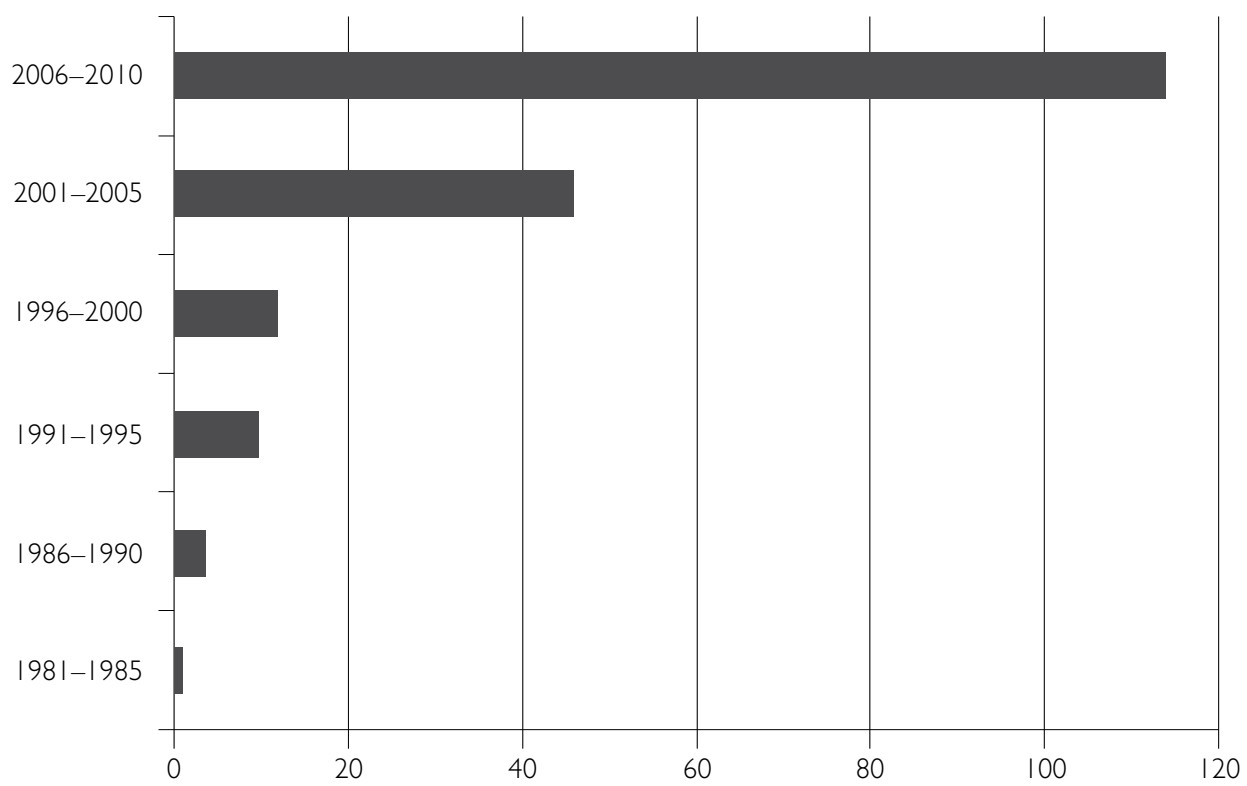

\section{Country}

Publications emanated largely from research conducted in the United States $(67 ; 36 \%)$, Canada (62; 33\%), the United Kingdom $(20 ; 11 \%)$ and Australia (19; 10\%), with additional 
contributions from researchers located in the Netherlands, Sweden, Germany, Iceland, France, Austria, Finland, New Zealand and China.

\section{Research approach and research design}

Of the 187 publications, 59 (32\%) were categorized as discussion papers. Another 51 studies $(27 \%)$ were classified as utilizing quantitative research methodologies. These included 22 studies (43\% of the quantitative group) described as randomized controlled trials, two of which were designated to be cluster-randomized controlled trials. Other research designs found in the quantitative group included non-randomized controlled trials, pre-post trials, trials with waiting list controls and cross-sectional surveys.

There were 51 (27\%) studies classified as qualitative in research approach. These included 26 studies (50\% of the qualitative group) that were described as utilizing a participatory action research design. Additional descriptors for qualitative studies included case studies, whereas others described the use of focus group, interview-based or thematic analysis methods.

There were 36 publications (19\%) that were classified as "other" in their research approach. These included 13 publications (36\% of the "other" group) classified as reviews and 10 studies (28\% of the "other" group) classified as using mixed-methods research designs (i.e., combining both quantitative and qualitative research approaches).

\section{Type of exchange and groups}

Of the total publications, 115 (62\%) addressed KT between two stakeholder groups, or dyads. More than half of those studies (62; 54\% of those classified as dyads) addressed KT between researchers and providers. Another 24 studies (21\% of those classified as dyads) addressed KT between PWLE and providers.

A total of 61 publications (33\%) were found to involve KT across three or more stakeholder groups, or polyads. These publications addressed KT across various combinations of stakeholder groups. The most frequent group combinations were PWLE-provider-researcher ( $9 ; 15 \%$ of polyads) and provider-policy maker-researcher $(8 ; 13 \%$ of polyads).

There were 11 publications (6\%) that appeared to focus on KT within one stakeholder group, described in Table 1 as "within group." Of these, 8 (73\% of those classified as "within group") focused on KT within groups of providers.

\section{Discussion and Conclusion}

Over the past decade, we found an exponential increase in the number of publications focused on KT in mental health. This increase appears to be consistent with growing interest in and awareness of this topic among the research community (e.g., OMHAKEN 2011).

The large proportion of publications (42\%) that were found to be of Canadian origin may be related to the endorsement of the importance of KT by Canadian research organizations such as the Canadian Health Services Research Foundation (Lomas 2000) and the Canadian Institutes of Health Research (Graham and Tetroe 2009). 
The publications we identified covered a wide range of research approaches and designs. A large proportion (59\%) were discussion papers, often advocating for the importance of KT in mental health services or advancing theoretical frameworks. However, there were also sizeable numbers of studies that applied qualitative, quantitative and mixed-method designs.

Papers that addressed the provider-researcher dyad tended to examine the effectiveness of efforts to change knowledge or behaviour among healthcare providers through KT interventions, often using an implementation science paradigm. Of these, only five studies report no improvement (Azocar et al. 2003; Goldberg et al. 1998; Lin et al. 2001; Ray et al. 1987; Thompson et al. 2000). The remaining studies indicate that carefully planned KT produces meaningful improvement in knowledge and practice regarding care for mental health problems (e.g., Avorn et al. 1992; Badger et al. 1991; Baker et al. 2001; B Brown et al. 2000; Gask et al. 1998; Gerrity et al. 1999; Hannaford et al. 1996; Mason and Freemantle 1999; Naismith et al. 2001; Ray et al. 1987; Rutz et al. 1992; van Eijk et al. 2001; Worrall et al. 1999). It is worth highlighting several Canadian studies:

- Barwick and colleagues (2009) randomly assigned mental health clinicians to a community of practice $(\mathrm{CoP})$ or practice as usual $(\mathrm{PaU})$ condition to support training in a newly implemented level of functioning outcome measurement tool. Practitioners in the CoP condition demonstrated greater use of the tool in practice, better content knowledge and greater satisfaction with implementation supports than $\mathrm{PaU}$ participants.

- Chagnon and colleagues (2008) helped develop a community of practice for researchers and providers working in the field of suicide prevention. Over a one-year period, a series of interventions designed to facilitate KT between members were carried out; several platforms (meetings, websites, listservs, online discussions and forums) allowed members to appraise their practices and integrate new knowledge on suicide prevention-related issues. The study by Chagnon and colleagues (2008) examined a CoP that was largely virtual in nature, whereas the study by Barwick and colleagues (2009) addressed an in vivo (face-to-face) CoP.

- In a study by Worrall and colleagues (1999), family physicians were randomly assigned to a three-hour, case-based educational session on clinical practice guidelines (CPGs) for depression and access to a psychiatrist for consultation or to a control group that received the CPG without the educational session or consultation. While there was no difference in rates of diagnosis or prescription, more patients in the intervention group were taking antidepressants at six-month follow-up, and referrals to psychiatry were slightly higher for this group.

- Bilsker and colleagues (2008) studied implementation of a one-hour educational session outlining interventions for depression and risky alcohol use and delivered to a sample of 85 family physicians. The interventions used a supported self-management approach and included free patient access to appropriate self-management resources. The study initially evaluated physicians' implementation of these interventions over a two-month period. Physician uptake of the depression intervention was significantly greater than uptake of the risky drinking intervention (32\% versus $10 \%$ ). A follow-up at six months 
post-training (depression intervention only) demonstrated fairly good maintenance of intervention delivery.

In addition to these Canadian studies cited above, research studies conducted in the United States and other countries have also examined KT interventions that seek to enhance primary mental healthcare services and improve outcomes of common mental health problems, primarily depressive disorders (e.g., Brown et al. 2000; Gerrity et al. 1999; Hodges et al. 2001; Naismith et al. 2001). More "active" KT approaches, such as implementation and dissemination activities described by Lomas (1993), have been found to be more effective in producing behavioural change and improved outcomes when compared with "passive" approaches described by Lomas (1993) as diffusion, such as mailing out clinical guidelines to primary care physician offices or posting information on a physician association website. A study in the United Kingdom (Baker et al. 2001) randomized family physicians to an intervention group that received a tailored implementation designed to assist them in adopting new clinical guidelines for treatment of depression. They were found to have adopted the new practices, and their patients with depression had better outcomes than those treated by a control group of family physicians who were notified of the new guidelines but did not receive the targeted $\mathrm{KT}$ implementation efforts.

Of the 115 studies classified as dyadic knowledge exchange, 24 (21\%) involved interactions between the PWLE-Provider dyad. KT between these two groups, in the form of shared decision-making, has become an increasingly common focus within the literature. As described by Drake and colleagues (2009):

The healthcare provider (often a team of professionals) brings expertise in understanding the medical problem, the possible interventions and the potential benefits and risks of alternatives. The patient (often assisted by family or support network members) brings expertise related to understanding the individual's values, goals, supports, and preferences.

Shared decision-making between PWLE and providers has also been studied through a series of randomized controlled trials examining the impacts of shared decision-making upon treatment satisfaction, quality of life and symptomatic improvement for schizophrenia, depression and substance use disorder (Hamann et al. 2006; Joosten et al. 2009; Loh et al. 2007; Malm et al. 2003; Priebe et al. 2007; Van Korff et al. 2003; Van Os et al. 2004). Overall, these studies indicate that shared decision-making enhances individuals' sense of involvement and likelihood of adhering to treatment. However, studies have not yet been able to demonstrate long-term benefits or improvements in function.

Participatory action research designs are well utilized in studies that involve PWLE. These approaches seek to incorporate the knowledge and skills of PWLE into various facets 
of research process, such as design, planning, implementation, analysis, reporting and dissemination. For example, Ross and colleagues (2003) worked with a group of young women to design and conduct focus groups with 48 adolescent girls across Ontario eliciting a set of themes helpful in planning interventions for adolescent depression. Participatory action research may be challenging to accomplish. McDaid (2009) worked with a group of participants and identified the following barriers to their equal participation: unequal cultural, physical, mental and economic resources, time and power; discrimination; and lack of respect for their experiential knowledge and emotional expression. Considerable dedication and commitment is often required to accomplish effective participatory action research.

Most publications concerning KT between the researcher-policy maker dyad comprise theoretical discussions of key issues affecting KT between these stakeholder groups. The lack of evaluative research has been identified by Mitton and colleagues (2008) as a major problem:

It is not hard to find opinion pieces and anecdotal reports about how to do KTE [knowledge transfer and exchange], but a limited reporting of actual KTE implementation and even more limited formal evaluation leaves those seeking to develop their own KTE efforts at a loss for evidence based strategies.

Some empirical studies of mental health-related KT between researchers and policy makers have been conducted in Canada, using qualitative methods and gathering data from focus groups or through stakeholder interviews:

- Waddell and colleagues (2007) report results of comprehensive interviews with researchers and funding decision-makers in order to identify barriers to KT in children's mental health policy efforts. Identified barriers included researchers' lack of motivation to maximize KT and neglect of strategies to foster knowledge uptake.

- Goering and colleagues (2003) present a case study of a project to enhance KT between researchers and policy makers by partnership between a research unit and a public agency for mental health policy. This included regular meetings between researchers and policy makers, initiation of interactive research projects and collaborative dissemination of research findings. The formalization of a structured agreement with shared activities and commitments provides an example of organizational linkage and exchange as described by Lomas (2000). A framework is shared that may be used by others seeking to build stable and mutually beneficial organizational relationships.

A number of studies address KT between researchers and the general public. Mental health-related $\mathrm{KT}$ in this domain generally involves the translation of scientific evidence via social marketing campaigns, websites, various communications media (including social media) to the general population, often seeking to increase mental health literacy. A recent Canadian 
report concluded that although Canadians are found to have a relatively high level of mental health literacy compared to citizens of other countries, "[s]tigma and discrimination toward persons with mental illness continue to be problematic in Canadian society" (CAMIMH 2008).

There is an informative empirical literature concerning the effectiveness of interventions to enhance the mental health literacy of general populations (e.g., Jorm et al. 2003; Wright et al. 2006; Goldney and Fisher 2008). Perhaps the most ambitious attempt to increase mental health literacy at a population level was the beyondblue program in Australia, which focused on depression awareness and beliefs. This extensive national campaign, undertaken over several years, was associated with improvements in knowledge by the general public about depression and its remediation (Jorm et al. 2005).

A notable group of publications $(61 ; 33 \%)$ was concerned with the exchange of knowledge among multiple stakeholders. Most of these publications report on qualitative investigation of KT among participant groups. We referred to these studies as polyadic, emphasizing their inclusion of multiple types of participants (i.e., polyads) in the KT process. The following are three Canadian examples:

- Westhues and colleagues (2008) sought to improve mental health service delivery to people of diverse cultural backgrounds. They engaged community members of various cultural and linguistic groups, service providers, governmental organizations and researchers. Working groups facilitated the concentration of multiple types of knowledge, subsequently exchanged among groups. This approach allowed a diverse group of stakeholders to create a framework for improving mental healthcare across cultural-linguistic communities.

- McGrath and colleagues (2009) applied an "integrated KT" strategy (i.e., engaging all stakeholders in KT at the beginning of the project and keeping them intimately involved throughout) to the implementation of a distance-based children's mental health program. They involved a wide range of participants (e.g., physicians, policy makers, service users and others) in all project phases, enabling ongoing KT among diverse stakeholder groups. The authors concluded that the integrated KT strategy was effective "in supporting the transfer of this research project into clinical practice" (McGrath et al. 2009: 30).

- Perreault and colleagues $(2005,2009)$ initiated a cross-training program for providers from different networks or sectors (e.g., mental health, substance use, police) who interact with people with concomitant mental health and substance use problems. Providers led or engaged in various training activities, including joint workshops and meetings, researcher presentations and staff rotations. Aiming to improve coordination and integration of care, the program also promoted shared knowledge, understanding and feelings of interdependence among participants.

A small but intriguing cluster of publications focus upon $\mathrm{KT}$ at the organizational rather than individual level (Isett and Provan 2004; Morrissey et al. 1997, 2005; Provan et al. 2005; Provan and Milward 1995, 2001). This type of research has been referred to as network analysis: 
Network analysis allows for the examination and comparison of relationships between two organizations (dyads), among clusters or cliques of organizations, and among all of the organizations comprised by the network. (Provan et al. 2005: 605)

For example, Provan and Milward (1995) conducted a network analysis of four community mental health systems engaged in integrated service delivery, each consisting of a network of mental health service providers, social service providers and funding agencies. Networks were evaluated for the strength of linkage and exchange among organizations and effectiveness of service delivery. The researchers found that network effectiveness could be explained by various structural and contextual factors, such as the degree of integration and stability that exists within the network.

Relevant publications tended to fall into a number of different "pockets" or thematic areas within the scientific literature. These include implementation science, community-based and participatory-action research, shared decision-making studies, mental health literacy research, network analysis and studies directly addressing KT. This full range of relevant literature is scattered across a variety of disparate research themes that are not usually assembled or considered together.

Overall, there appears to be a robust interest in addressing KT in relation to mental health issues, with strong representation from Canadian stakeholder groups. This appears to have been a burgeoning area of study in recent years, and valuable information is now becoming available to help guide activities and efforts aimed at improving various facets of the mental health "system."

In the field of mental health, KT approaches appear to be undergoing a paradigm shift. Researchers continue to play a unique role in generating, disseminating and exchanging valuable scientific knowledge, but they have been joined by other key stakeholder groups such as policy makers, healthcare providers, family members and, importantly, people with lived experience of mental health problems. The value of experiential knowledge is better recognized (CaronFlinterman et al. 2005), and KT is better understood to be a diverse and sometimes complex set of steps that involve the participation of diverse actors. This shift has been described explicitly in a framework document of the Canadian Mental Health Association (n.d.) and may prove to have far-reaching effects upon mental health research, policy and practice.

\section{Limitations}

Limitations of the current scoping review include the possibility that some relevant publications may not have been located despite use of systematic search methods and iterative steps to minimize omissions. Application of the inclusion criteria used in selecting studies for review may have been imperfect despite high ratings on tests of inter-rater reliability, and there was inevitable subjectivity in the decision-making process when considering whether studies were or were not relevant. The current findings cannot be assumed to be valid in relation to low-income countries, as we excluded studies that focused on KT in such regions. In addition, we recognize the possibility that the results of this process may not be truly comprehensive, 
owing to the ambiguity surrounding conceptualizations of $\mathrm{KT}$ in combination with the relative isolation of some KT research areas.

Correspondence may be directed to: Dr. Elliot M. Goldner, Professor, Faculty of Health Sciences, Simon Fraser University, Harbour Centre, 2400 - 515 West Hastings St., Vancouver, BC V6B 5K3; tel. 778-782-5148; e-mail: egoldner@sfu.ca.

\section{REFERENCES}

Arksey, H. and L. O'Malley. 2005. “Scoping Studies: Towards a Methodological Framework." Social Research Methods 8: 19-31.

Avorn, J., S.B. Soumerai, D.E. Everitt et al. 1992. "A Randomized Trial of a Program to Reduce the Use of Psychoactive Drugs in Nursing Homes." New England Journal of Medicine 327: 168-73.

Azocar, F., B. Cuffel, W. Goldman and L. McCarter. 2003."The Impact of Evidence-Based Guideline Dissemination for the Assessment and Treatment of Major Depression in a Managed Behavioral Health Care Organization." Journal of Behavioral Health Services and Research 30(1): 109-18.

Badger, T.A., M.H. Mishel, L.J. Biocca et al. 1991. “Depression Assessment and Management: Evaluating a Community-Based Mental Health Training Program for Nurses." Public Health Nursing 8: 170-75.

Baker, R., S. Reddish, N. Robertson, H. Hearnshaw and B. Jones. 2001. "Randomised Controlled Trial of Tailored Strategies to Implement Guidelines for the Management of Patients with Depression in General Practice." British Journal of General Practice 51(470): 737-41.

Barwick, M., J. Peters and K. Boydell. 2009. “Getting to Uptake: Do Communities of Practice Support the Implementation of Evidence-Based Practice?" Journal of the Canadian Academy of Child E Adolescent Psychiatry 18(1): 16-29.

Bilsker, D., J. Anderson, J. Samra, E. Goldner and D. Streiner. 2008. “Behavioural Interventions in Primary Care: An Implementation Trial." Canadian Journal of Community Mental Health 27: 179-89.

Brown, J.B., D. Shye, B.H. McFarland, G.A. Nichols, J.P. Mullooly and R.E. Johnson. 2000. “Controlled Trials of CQI and Academic Detailing to Implement a Clinical Practice Guideline for Depression." Joint Commission Journal on Quality Improvement 26: 39-54.

Canadian Alliance on Mental Illness and Mental Health (CAMIMH). 2008. "National Integrated Framework for Enhancing Mental Health Literacy in Canada: Final Report." Retrieved October 2, 2011. <http://www.camimh. ca/files/literacy/CAMIMH\%20MHL\%20National\%20Integrated\%20Framework\%20July\%202008.pdf>.

Canadian Mental Health Association. n.d. Knowledge Exchange: A Framework for Action by the Canadian Mental Health Association. Ottawa: Author.

Caron-Flinterman, J.F., J.E.W. Broerse and J.F.G. Bunders. 2005."The Experiential Knowledge of Patients: A New Resource for Biomedical Research?" Social Science \& Medicine 60: 2575-84.

Chagnon, F., J. Houle, M. Daigle, B.L. Mishara and C. Bardon. 2008. “Application des connaissances scientifiques en prévention du suicide : vérification d'une stratégie fondée sur la communauté de pratique." Frontières 21(1): 90-97.

Dobbins, M., S.E. Hanna, D. Ciliska, S. Manske, R. Cameron, S.L. Mercer et al. 2009. “A Randomized Controlled Trial Evaluating the Impact of Knowledge Translation and Exchange Activities." Implementation Science 4(61).

Drake, R.E., D. Cimpean and W.C. Torrey. 2009. "Shared Decision-Making in Mental Health: Prospects for Personalized Medicine." Dialogues in Clinical NeuroScience 11: 455-63.

Driedger, S.M., A. Kothari, I.D. Graham, E. Cooper, E.J. Crighton, M. Zahab et al. 2010. “If You Build It, They Still May Not Come: Outcomes and Process of Implementing a Community-Based Integrated Knowledge Translation Mapping Innovation." Implementation Science 16(5): 47.

Gagnon, M. 2009. “Knowledge Dissemination and Exchange of Knowledge." In S. Straus, J. Tetroe and I.D. Graham, eds., Knowledge Translation in Healthcare: Moving from Evidence to Practice. Oxford: Wiley-Blackwell. 
Gask, L., T. Usherwood, H. Thompson et al. 1998. "Evaluation of a Training Package in the Assessment and Management of Depression in Primary Care." Medical Education 32: 190-98.

Gerrity, M.S., S.A. Cole, A.J. Dietrich and J.E. Barrett. 1999. "Improving the Recognition and Management of Depression: Is There a Role for Physician Education?" Journal of Family Practice 48(12): 949-57.

Goering, P., D. Buterill, N. Jacobson and D. Sturtevant. 2003. “Linkage and Exchange at the Organizational Level: A Model of Collaboration Between Research and Policy." Journal of Health Services Research E Policy 8: 14-19.

Goldberg, H.I., E.H. Wagner, S.D. Fihn, D.P. Martin, C.R. Horowitz, D.B. Christensen et al. 1998. “A Randomized Controlled Trial of CQI Teams and Academic Detailing: Can They Alter Compliance with Guidelines?" Joint Commission Journal on Quality Improvement 24: 130-42.

Goldney, R.D. and L.J. Fisher. 2008. "Have Broad-Based Community and Professional Education Programs Influenced Mental Health Literacy and Treatment Seeking of Those with Major Depression and Suicidal Ideation?" Suicide and Life-Threatening Behavior 38(2): 129-42.

Graham, I.D. and J.M. Tetroe. 2009. “Getting Evidence into Policy and Practice: Perspective of a Health Research Funder." Journal of the Canadian Academy of Child \& Adolescent Psychiatry 18(1): 46-50.

Grimshaw, J.M., L. Shirran, R. Thomas, G. Mowatt, C. Fraser, L. Bero et al. 2001. "Changing Provider Behavior: An Overview of Systematic Reviews of Interventions.” Medical Care 39(8 Suppl. 2): 112-45.

Hamann, J., B. Langer, V. Winkler, R. Busch, R. Cohen, S. Leucht et al. 2006. “Shared Decision Making for Inpatients with Schizophrenia." Acta Psychiatrica Scandinavica 114: 265-73.

Hannaford, P.C., C. Thompson and M. Simpson. 1996. "Evaluation of an Educational Program to Improve the Recognition of Psychological Illness by General Practitioners." British Journal of General Practice 46: 333-37.

Hodges, B., C. Inchand and I. Silver. 2001. "Improving the Psychiatric Knowledge, Skills, and Attitudes of Primary Care Physicians, 1950-2000: A Review." American Journal of Psychiatry 158: 1579-86.

Isett, K.R. and K.G. Provan. 2004. "The Evolution of Interorganizational Network Relationships Over Time: Does Sector Matter?" Journal of Public Administration Research and Theory 15: 149-65.

Jacobson, N., D. Butterill and P. Goering. 2003. “Development of a Framework for Knowledge Translation: Understanding User Context." Journal of Health Services Research E Policy 8: 94-99.

Joosten, E.A., C.A. de Jong, G.H. de Weert-van Oene, T. Sensky and C.P. van der Staak. 2009. "Shared DecisionMaking Reduces Drug Use and Psychiatric Severity in Substance-Dependent Patients." Psychotherapy and Psychosomatics 78: 245-53.

Jorm, A.F., H. Christiansen and K.M. Griffiths. 2005. “The Impact of beyondblue: The National Depression Initiative on the Australian Public's Recognition of Beliefs about Treatments." Australian E New Zealand Journal of Psychiatry 39(4): 248-54.

Jorm, A.F., K.M. Griffiths, H. Christensen, A.E. Korten, R.A. Parslowand and B. Rodgers. 2003. “Providing Information About the Effectiveness of Treatment Options to Depressed People in the Community: A Randomized, Controlled Trial of Effects on Mental Health Literacy, Help-Seeking and Symptoms." Psychological Medicine 33: 1071-79.

Lavis, J.N., J. Lomas, M. Hamid and N.K. Sewankambo. 2006. “Assessing Country-Level Efforts to Link Research to Action." Bulletin of the World Health Organization 84(8): 620-28.

Légaré, F., S. Ratté, D. Stacey, J. Kryworuchko, K. Gravel, I.D. Graham et al. 2010. “Interventions for Improving the Adoption of Shared Decision Making by Healthcare Professionals." Cochrane Database System Review 5: CD006732.

Levac, D., H. Colquhoun and K.K. O’Brien. 2010. “Scoping Studies: Advancing the Methodology." Implementation Science 5: 69.

Lin, E.H., G.E. Simon, D.J. Katzelnick and S.D. Pearson. 2001. “Does Physician Education on Depression Management Improve Treatment in Primary Care?” Journal of General Internal Medicine 16: 614-19.

Loh, A., D. Simon, C.E. Wills, L. Kriston, W. Niebling and M. Harter. 2007. “The Effects of a Shared DecisionMaking Intervention in Primary Care of Depression: A Cluster-Randomized Controlled Trial." Patient Education and Counseling 67: 324-32. 


\section{Knowledge Translation in Mental Health}

Lomas, J. 1993. "Diffusion, Dissemination and Implementation: Who Should Do What?" Annals of the New York Academy of Science 703: 226-35.

Lomas, J. 2000. “Using 'Linkage and Exchange' to Move Research into Policy at a Canadian Foundation." Health Affairs 19(3): 236-40.

Malm, U., B. Ivarsson, P. Allebeck and I. Falloon. 2003. “Integrated Care in Schizophrenia: A Two-Year Randomized Controlled Study of Two Community-Based Treatment Programs." Acta Psychiatrica Scandinavica 107: 415-23.

Mason, J. and N. Freemantle. 1999."The Effect of the Distribution of Effective Healthcare Bulletins on Prescribing Selective Serotonin Reuptake Inhibitors in Primary Care." Health Trends 30: 120-25.

McDaid, S. 2009. "An Equality of Condition Framework for User Involvement in Mental Health Policy and Planning: Evidence from Participatory Action Research." Disability E Society 24(4): 461-74.

McGrath, P.J., P. Lingley-Pottie, D.J. Emberly, C. Thurston and C. McLean. 2009.“Integrated Knowledge

Translation in Mental Health: Family Help as an Example." Journal of the Canadian Academy of Child E Adolescent Psychiatry 18: 30-37.

Mitton, C., C.E. Adair, E. McKenzie, S. Patten and B.W. Perry. 2007.“Knowledge Transfer and Exchange: Review and Synthesis of the Literature." Milbank Quarterly 85: 729-68.

Mitton, C., C.E. Adair, E. McKenzie, S. Patten, B.W. Perry and N. Smith. 2008 (June).“Knowledge Transfer and Exchange (KT): A Systematic Review, Key Informant Interviews and Design of a KT Strategy." In D. Hailey, J. Grimshaw, M. Eccles et al., eds., Effective Dissemination of Findings from Research: A Compilation of Essays. Edmonton: Institute of Health Economics.

Morrissey, J.P., M.C. Johnsen and M.O. Calloway. 1997.“Evaluating Performance and Change in Mental Health Systems Serving Children and Youth: An Interorganizational Network Approach." Journal of Behavioral Health Services and Research 24(1): 4-22.

Morrissey, J.P., A.R. Ellis, M. Gatz, H. Amaro, B.G. Reed, A. Savage et al. 2005. “Outcomes for Women with Co-Occurring Disorders and Trauma: Program and Person-Level Effects." Journal of Substance Abuse Treatment 28(2): 121-33.

Naismith, S.L., I.B. Hickie, E.M. Scott and T.A. Davenport. 2001. “Effects of Mental Health Training and Clinical Audit on General Practitioners' Management of Common Mental Disorders." Medical Journal of Australia 175: S42-47.

Ontario Mental Health and Addictions Knowledge Exchange Network (OMHAKEN) on behalf of Creating Together partners. 2011. Co-Creating a Mental Health and Addictions Research Agenda for Ontario. Final Report. Toronto: Centre for Addiction and Mental Health.

Perreault, M., J.P. Bonin, R. Veilleux, G. Alary and I. Ferland. 2005.“Expérience de formation croisée dans un contexte d'intégration des services en réseau dans le sud-ouest de Montréal." Revue Canadienne de Santé Mentale Communautaire 24(1): 35-49.

Perreault, M., D. Wiethaueper, N. Perreault, J.P. Bonin, T. Brown and H. Brunaud. 2009."Meilleures pratiques et formation dans le contexte du continuum des services en santé mentale et en toxicomanie : le programme de formation croisée du sud-ouest de Montréal." Santé Mentale au Québec 34(1): 143-60.

Priebe, S., R. McCabe, J. Bullenkamp, L. Hansson, C. Lauber and R. Martinez-Leall. 2007. “Structured PatientClinician Communication and 1-Year Outcome in Community Mental Healthcare." British Journal of Psychiatry 191: 420-26.

Provan, K.G. and H.B. Milward. 1995. "A Preliminary Theory of Interorganizational Network Effectiveness: A Comparative Study of Four Community Mental Health Systems." Administrative Science Quarterly 40(1): 1-33.

Provan, K.G. and H.B. Milward. 2001. “Do Networks Really Work? A Framework for Evaluating Public-Sector Organizational Networks." Public Administration Review 61(4): 414-23.

Provan, K.G., M.A. Veazie, L.K. Staten and N.I. Teufel-Shone. 2005."The Use of Network Analysis to Strengthen Community Partnerships." Public Administration Review 65: 603-14.

Ray, W.A., D.G. Blazer, W. Schaffner and C.F. Federspiel. 1987.“Reducing Drug Prescribing for Nursing Home Patients: A Controlled Trial of the Effect of an Educational Visit." American Journal of Public Health 77(11): $1448-50$. 
Ross, E., A. Ali and B. Toner. 2003. “Investigating Issues Surrounding Depression in Adolescent Girls Across Ontario: A Participatory Action Research Project." Canadian Journal of Community Mental Health 22(1): 55-68.

Rutz, W., L. von Knorring and J. Walinder. 1992. "Long-Term Effects of an Educational Program for General Practitioners Given by the Swedish Committee for the Prevention and Treatment of Depression." Acta Psychiatrica Scandinavica 85: 83-88.

Straus, S.E., J. Tetroe and I.D. Graham. 2009. “Defining Knowledge Translation.” Canadian Medical Association Journal 181: 165-68.

Thompson, C., A.L. Kinmonth, L. Stevens et al. 2000."Effects of a Clinical Practice Guideline and PracticeBased Education on Detection and Outcome of Depression in Primary Care: Hampshire Depression Project Randomised Controlled Trial." The Lancet 355(9199): 185-91.

van Eijk, M.E.C., J. Avorn, A.J. Porsius et al. 2001."Reducing Prescribing of Highly Anticholinergic

Antidepressants for Elderly People: Randomized Trial of Group versus Individual Academic Detailing." British Medical Journal 322: 654-57.

Van Korff, M., W. Katon, C. Rutter, E. Ludman, G. Simon, E. Lin et al. 2003."Effect on Disability Outcomes of a Depression Relapse Prevention Program.” Psychosomatic Medicine 65: 938-43.

Van Os, J., A.C. Altamura, J. Bobes, J. Gerlach, J.S. Hellewell, S. Kasper et al. 2004. "Evaluation of the Two-Way Communication Checklist as a Clinical Intervention: Results of a Multinational, Randomised Controlled Trial." British Journal of Psychiatry 184: 79-83.

Waddell, C., J.N. Lavis, J. Abelson, J. Lomas, C.A. Shepherd, T. Bird-Gayson et al. 2005. “Research Use in Children's Mental Health Policy in Canada: Maintaining Vigilance Amid Ambiguity." Social Science E Medicine 61(8): 1649-57.

Waddell, C., C.A. Shepherd, J.N. Lavis, J.N. Lomas, J. Abelson and T. Bird-Gayson. 2007.“Balancing Rigour and Relevance: Researchers' Contributions to Children's Mental Health in Canada." Evidence and Policy 3(2): 181-95.

Westhues, A., J. Ochocka, N. Jacobson, L. Simich, S. Maiter, R. Janzen et al. 2008. “Developing Theory from Complexity: Reflections on a Collaborative Mixed-Methods Participatory Action Research Study." Qualitative Health Research 18: 701-17.

Worrall, G., J. Angel, P. Chaulk, C. Clarke and M. Robbins. 1999. “Effectiveness of an Educational Strategy to Improve Family Physicians' Detection and Management of Depression: A Randomized Controlled Trial.” Canadian Medical Association Journal 161: 37-40.

Wright, A., P.D. McGorry, M.G. Harris, A.F. Jorm and K. Pennell. 2006. “Development and Evaluation of a Youth Mental Health Community Awareness Campaign: The Compass Strategy" BMC Public Health 6: 215. 


\section{Knowledge Translation in Mental Health: A Scoping Review Échange de connaissances en santé mentale : analyse de la situation}

ELLIOT M. GOLDNER, VICTORIA JEFFRIES, DAN BILSKER, EMILY JENKINS, MATTHEW MENEAR AND LISA PETERMANN

\begin{tabular}{|c|c|c|c|c|c|c|c|c|}
\hline Authors & Year & Title & Publication & Country & $\begin{array}{l}\text { Research } \\
\text { approach }\end{array}$ & $\begin{array}{l}\text { Research } \\
\text { design }\end{array}$ & $\begin{array}{l}\text { Type of } \\
\text { exchange }\end{array}$ & Groups \\
\hline $\begin{array}{l}\text { Byrnes, E. \& } \\
\text { Johnson, J.H. }\end{array}$ & 1981 & $\begin{array}{l}\text { Change technology and } \\
\text { the implementation of } \\
\text { automation in mental } \\
\text { healthcare settings }\end{array}$ & $\begin{array}{l}\text { Behavior Research } \\
\text { Methods 13(4): 573-80 }\end{array}$ & USA & Discussion & Discussion & Dyad & $\begin{array}{l}\text { Provider- } \\
\text { Policy maker }\end{array}$ \\
\hline $\begin{array}{l}\text { Backer, T.E., } \\
\text { Liberman, R.P., } \\
\text { Kuehnel, T.G. }\end{array}$ & 1986 & $\begin{array}{l}\text { Dissemination } \\
\text { and adoption of } \\
\text { innovative psychosocial } \\
\text { interventions }\end{array}$ & $\begin{array}{l}\text { Journal of Consulting and } \\
\text { Clinical Psychology } 54(1) \text { : } \\
\text { |। |-18 }\end{array}$ & USA & Discussion & Discussion & Polyad & $\begin{array}{l}\text { Provider- } \\
\text { Policy maker- } \\
\text { Researcher }\end{array}$ \\
\hline $\begin{array}{l}\text { Ray, W.A., } \\
\text { Blazer, D.G., } \\
\text { Schaffner, W., } \\
\text { Federspiel, } \\
\text { C.F. }\end{array}$ & 1987 & $\begin{array}{l}\text { Reducing antipsychotic } \\
\text { drug prescribing for } \\
\text { nursing home patients: } \\
\text { a controlled trial of the } \\
\text { effect of an educational } \\
\text { visit }\end{array}$ & $\begin{array}{l}\text { American Journal of Public } \\
\text { Health 77: | } 448-50\end{array}$ & USA & Quantitative & $\begin{array}{l}\text { Non- } \\
\text { randomized } \\
\text { controlled } \\
\text { trial }\end{array}$ & Dyad & $\begin{array}{l}\text { Provider- } \\
\text { Researcher }\end{array}$ \\
\hline $\begin{array}{l}\text { Tremblay, } \\
\text { M.-A. \& } \\
\text { Poirier, C. }\end{array}$ & 1989 & $\begin{array}{l}\text { La Construction } \\
\text { culturelle de la recherche } \\
\text { psychosociale en } \\
\text { santé mentale : les } \\
\text { enjeux scientifiques et } \\
\text { sociopolitiques }\end{array}$ & $\begin{array}{l}\text { Santé Mentale au Québec } \\
\text { |4(I): I 1-34 }\end{array}$ & Canada & Discussion & Discussion & Dyad & $\begin{array}{l}\text { Provider- } \\
\text { Researcher }\end{array}$ \\
\hline $\begin{array}{l}\text { Andersen, } \\
\text { S.M. \& } \\
\text { Harthorn, } \\
\text { B.H. }\end{array}$ & 1990 & $\begin{array}{l}\text { Changing the psychiatric } \\
\text { knowledge of primary } \\
\text { care physicians: the } \\
\text { effects of a brief } \\
\text { intervention on clinical } \\
\text { diagnosis and treatment }\end{array}$ & $\begin{array}{l}\text { General Hospital } \\
\text { Psychiatry 12: 177-90 }\end{array}$ & USA & Quantitative & $\begin{array}{l}\text { Quasi- } \\
\text { experimental }\end{array}$ & Dyad & $\begin{array}{l}\text { Provider- } \\
\text { Researcher }\end{array}$ \\
\hline $\begin{array}{l}\text { Badger, T.A., } \\
\text { Mishel, M.H., } \\
\text { Biocca, L.J. } \\
\text { et al. }\end{array}$ & |99| & $\begin{array}{l}\text { Depression assessment } \\
\text { and management: } \\
\text { evaluating a community- } \\
\text { based mental health } \\
\text { training program for } \\
\text { nurses }\end{array}$ & $\begin{array}{l}\text { Public Health Nursing 8: } \\
\text { 170-75 }\end{array}$ & USA & Quantitative & Pre-post & Dyad & $\begin{array}{l}\text { Provider- } \\
\text { Researcher }\end{array}$ \\
\hline $\begin{array}{l}\text { Steadman, } \\
\text { H.J. }\end{array}$ & 1992 & $\begin{array}{l}\text { Boundary spanners: a } \\
\text { key component for the } \\
\text { effective interactions of } \\
\text { the justice and mental } \\
\text { health systems }\end{array}$ & $\begin{array}{l}\text { Law and Human Behavior } \\
\text { 16(1): 75-87 }\end{array}$ & USA & Discussion & Discussion & Dyad & $\begin{array}{l}\text { Provider- } \\
\text { Other }\end{array}$ \\
\hline $\begin{array}{l}\text { Avorn, J., } \\
\text { Soumerai, } \\
\text { S.B., Everitt, } \\
\text { D.E. et al. }\end{array}$ & 1992 & $\begin{array}{l}\text { A randomized trial of a } \\
\text { program to reduce the } \\
\text { use of psychoactive drugs } \\
\text { in nursing homes }\end{array}$ & $\begin{array}{l}\text { New England Journal of } \\
\text { Medicine 327: 168-73 }\end{array}$ & USA & Quantitative & $\begin{array}{l}\text { Randomized } \\
\text { controlled } \\
\text { trial }\end{array}$ & Dyad & $\begin{array}{l}\text { Provider- } \\
\text { Researcher }\end{array}$ \\
\hline
\end{tabular}


Elliot M. Goldner et al.

\begin{tabular}{|c|c|c|c|c|c|c|c|c|}
\hline Authors & Year & Title & Publication & Country & $\begin{array}{l}\text { Research } \\
\text { approach }\end{array}$ & $\begin{array}{l}\text { Research } \\
\text { design }\end{array}$ & $\begin{array}{l}\text { Type of } \\
\text { exchange }\end{array}$ & Groups \\
\hline $\begin{array}{l}\text { Rutz, W., von } \\
\text { Knorring, L., } \\
\text { Walinder, J. }\end{array}$ & 1992 & $\begin{array}{l}\text { Long-term effects } \\
\text { of an educational } \\
\text { program for general } \\
\text { practitioners given by } \\
\text { the Swedish Committee } \\
\text { for the Prevention and } \\
\text { Treatment of Depression }\end{array}$ & $\begin{array}{l}\text { Acta Psychiatrica } \\
\text { Scandinavica 85: 83-88 }\end{array}$ & Sweden & Quantitative & Pre-post & Dyad & $\begin{array}{l}\text { Provider- } \\
\text { Researcher }\end{array}$ \\
\hline $\begin{array}{l}\text { Ray, W.A., } \\
\text { Taylor, J.A., } \\
\text { Meador, K.G. } \\
\text { et al. }\end{array}$ & 1993 & $\begin{array}{l}\text { Reducing antipsychotic } \\
\text { drug use in nursing } \\
\text { homes: a controlled trial } \\
\text { of provider education }\end{array}$ & $\begin{array}{l}\text { Archives of Internal } \\
\text { Medicine 153: 7|3-2| }\end{array}$ & USA & Quantitative & $\begin{array}{l}\text { Non- } \\
\text { randomized } \\
\text { controlled } \\
\text { trial }\end{array}$ & Dyad & $\begin{array}{l}\text { Provider- } \\
\text { Researcher }\end{array}$ \\
\hline $\begin{array}{l}\text { Clément, M., } \\
\text { Ouellet, F., } \\
\text { Coulombe, L. } \\
\text { et al. }\end{array}$ & 1995 & $\begin{array}{l}\text { Le partenariat de } \\
\text { recherche : éléments de } \\
\text { définition et ancrage dans } \\
\text { quelques études de cas }\end{array}$ & $\begin{array}{l}\text { Service Social 44(2): } \\
\text { | } 47-64\end{array}$ & Canada & Discussion & Discussion & Dyad & $\begin{array}{l}\text { Provider- } \\
\text { Researcher }\end{array}$ \\
\hline $\begin{array}{l}\text { de Burgh, S., } \\
\text { Mattick, R.P., } \\
\text { Donnelly, N. } \\
\text { et al. }\end{array}$ & 1995 & $\begin{array}{l}\text { A controlled trial of } \\
\text { educational visiting to } \\
\text { improve benzodiazepine } \\
\text { prescribing in general } \\
\text { practice }\end{array}$ & $\begin{array}{l}\text { Australian Journal of Public } \\
\text { Health 19: 142-48 }\end{array}$ & Australia & Quantitative & $\begin{array}{l}\text { Randomized } \\
\text { controlled } \\
\text { trial }\end{array}$ & Dyad & $\begin{array}{l}\text { Provider- } \\
\text { Researcher }\end{array}$ \\
\hline $\begin{array}{l}\text { Kendrick, } \\
\text { T., Burns T., } \\
\text { Freeling, P. }\end{array}$ & 1995 & $\begin{array}{l}\text { Randomised controlled } \\
\text { trial of teaching general } \\
\text { practitioners to carry out } \\
\text { structured assessments of } \\
\text { their long-term mentally } \\
\text { ill patients }\end{array}$ & $\begin{array}{l}\text { British Medical Journal } \\
311: 93-97\end{array}$ & UK & Quantitative & $\begin{array}{l}\text { Randomized } \\
\text { controlled } \\
\text { trial }\end{array}$ & Dyad & $\begin{array}{l}\text { Provider- } \\
\text { Researcher }\end{array}$ \\
\hline $\begin{array}{l}\text { Constantino, } \\
\text { V. \& Nelson, } \\
\text { G. }\end{array}$ & 1995 & $\begin{array}{l}\text { Changing relationships } \\
\text { between self-help } \\
\text { groups and mental health } \\
\text { professionals: shifting } \\
\text { ideology and power }\end{array}$ & $\begin{array}{l}\text { Canadian Journal of } \\
\text { Community Mental Health } \\
\text { 14(2): 55-70 }\end{array}$ & Canada & Qualitative & Focus groups & Polyad & $\begin{array}{l}\text { PWLE- } \\
\text { Family- } \\
\text { Provider }\end{array}$ \\
\hline $\begin{array}{l}\text { Hill, C.E. \& } \\
\text { Fraser, G.J. }\end{array}$ & 1995 & $\begin{array}{l}\text { Local knowledge and } \\
\text { rural mental health } \\
\text { reform }\end{array}$ & $\begin{array}{l}\text { Community Mental Health } \\
\text { Journal 3I(6): 553-68 }\end{array}$ & USA & Discussion & $\begin{array}{l}\text { Discussion, } \\
\text { participatory } \\
\text { action theory }\end{array}$ & Polyad & $\begin{array}{l}\text { PWLE- } \\
\text { Family- } \\
\text { Provider- } \\
\text { Policy maker- } \\
\text { Researcher }\end{array}$ \\
\hline $\begin{array}{l}\text { Hannaford, } \\
\text { P.C., } \\
\text { Thompson, } \\
\text { C., Simpson, } \\
\text { M. }\end{array}$ & 1996 & $\begin{array}{l}\text { Evaluation of an } \\
\text { educational programme } \\
\text { to improve the } \\
\text { recognition of } \\
\text { psychological illness by } \\
\text { general practitioners }\end{array}$ & $\begin{array}{l}\text { British Journal of General } \\
\text { Practice 46: 333-37 }\end{array}$ & UK & Quantitative & Pre-post & Dyad & $\begin{array}{l}\text { Provider- } \\
\text { Researcher }\end{array}$ \\
\hline Campbell, J. & 1996 & $\begin{array}{l}\text { Toward collaborative } \\
\text { mental health outcome } \\
\text { systems }\end{array}$ & $\begin{array}{l}\text { New Directions for } \\
\text { Mental Health Services } \\
\text { 71: 69-78 }\end{array}$ & USA & Discussion & Discussion & Dyad & $\begin{array}{l}\text { PWLE- } \\
\text { Provider }\end{array}$ \\
\hline
\end{tabular}


Knowledge Translation in Mental Health

\begin{tabular}{|c|c|c|c|c|c|c|c|c|}
\hline Authors & Year & Title & Publication & Country & $\begin{array}{l}\text { Research } \\
\text { approach }\end{array}$ & $\begin{array}{l}\text { Research } \\
\text { design }\end{array}$ & $\begin{array}{l}\text { Type of } \\
\text { exchange }\end{array}$ & Groups \\
\hline $\begin{array}{l}\text { Burns, T., } \\
\text { Millar, E., } \\
\text { Garland, C. } \\
\text { et al. }\end{array}$ & 1998 & $\begin{array}{l}\text { Randomised controlled } \\
\text { trial of teaching practice } \\
\text { nurses to carry out } \\
\text { structured assessments of } \\
\text { patients receiving depot } \\
\text { antipsychotic injections }\end{array}$ & $\begin{array}{l}\text { British Medical Journal } \\
\text { 48(437): | } 845-48\end{array}$ & UK & Quantitative & $\begin{array}{l}\text { Randomized } \\
\text { controlled } \\
\text { trial }\end{array}$ & Dyad & $\begin{array}{l}\text { Provider- } \\
\text { Researcher }\end{array}$ \\
\hline $\begin{array}{l}\text { Gask, L., } \\
\text { Usherwood, } \\
\text { T., Thompson, } \\
\text { H. et al. }\end{array}$ & 1998 & $\begin{array}{l}\text { Evaluation of a } \\
\text { training package in } \\
\text { the assessment and } \\
\text { management of } \\
\text { depression in primary } \\
\text { care }\end{array}$ & $\begin{array}{l}\text { Medical Education 32: } \\
\text { 190-98 }\end{array}$ & UK & Other & $\begin{array}{l}\text { Mixed } \\
\text { methods }\end{array}$ & Dyad & $\begin{array}{l}\text { Provider- } \\
\text { Researcher }\end{array}$ \\
\hline $\begin{array}{l}\text { Goldberg, } \\
\text { H.I., Wagner, } \\
\text { E.H. et al. }\end{array}$ & 1998 & $\begin{array}{l}\text { A randomized controlled } \\
\text { trial of CQI teams and } \\
\text { academic detailing: can } \\
\text { they alter compliance } \\
\text { with guidelines? }\end{array}$ & $\begin{array}{l}\text { Joint Commission Journal } \\
\text { on Quality Improvement } \\
\text { 24: 130-42 }\end{array}$ & USA & Quantitative & $\begin{array}{l}\text { Randomized } \\
\text { controlled } \\
\text { trial }\end{array}$ & Dyad & $\begin{array}{l}\text { Provider- } \\
\text { Researcher }\end{array}$ \\
\hline $\begin{array}{l}\text { Gerrity, M.S., } \\
\text { Cole, S.A., } \\
\text { Dietrich, A.J. } \\
\text { et al. }\end{array}$ & 1999 & $\begin{array}{l}\text { Improving the recognition } \\
\text { and management of } \\
\text { depression: is there } \\
\text { a role for physician } \\
\text { education? }\end{array}$ & $\begin{array}{l}\text { Journal of Family Practice: } \\
48(12): 949-57\end{array}$ & USA & Quantitative & $\begin{array}{l}\text { Randomized } \\
\text { controlled } \\
\text { trial }\end{array}$ & Dyad & $\begin{array}{l}\text { Provider- } \\
\text { Researcher }\end{array}$ \\
\hline $\begin{array}{l}\text { Mason, J. \& } \\
\text { Freemantle, } \\
\text { N. }\end{array}$ & 1999 & $\begin{array}{l}\text { The effect of the } \\
\text { distribution of Effective } \\
\text { Healthcare Bulletins on } \\
\text { prescribing selective } \\
\text { serotonin reuptake } \\
\text { inhibitors in primary care }\end{array}$ & Health Trends 30: 120-25 & UK & Quantitative & Pre-post & Dyad & $\begin{array}{l}\text { Provider- } \\
\text { Researcher }\end{array}$ \\
\hline $\begin{array}{l}\text { Worrall, G., } \\
\text { Angel, J., } \\
\text { Chaulk, P. } \\
\text { et al. }\end{array}$ & 1999 & $\begin{array}{l}\text { Effectiveness of an } \\
\text { educational strategy } \\
\text { to improve family } \\
\text { physicians' detection } \\
\text { and management } \\
\text { of depression: a } \\
\text { randomized controlled } \\
\text { trial }\end{array}$ & $\begin{array}{l}\text { Canadian Medical } \\
\text { Association Journal } 16 \mid(1) \text { : } \\
37-40\end{array}$ & Canada & Quantitative & $\begin{array}{l}\text { Randomized } \\
\text { controlled } \\
\text { trial }\end{array}$ & Dyad & $\begin{array}{l}\text { Provider- } \\
\text { Researcher }\end{array}$ \\
\hline $\begin{array}{l}\text { Poirier, M., } \\
\text { Larose, S., } \\
\text { Ste-Marie, F. } \\
\text { et al. }\end{array}$ & 1999 & $\begin{array}{l}\text { Splendeurs et misères de } \\
\text { la concertation locale en } \\
\text { santé mentale : un regard } \\
\text { du terrain }\end{array}$ & $\begin{array}{l}\text { Canadian Journal of } \\
\text { Community Mental Health } \\
\text { I8(2): 1 13-29 }\end{array}$ & Canada & Discussion & Discussion & Within group & $\begin{array}{l}\text { Provider- } \\
\text { Provider }\end{array}$ \\
\hline $\begin{array}{l}\text { Brown, J.B., } \\
\text { Shye, D. et al. }\end{array}$ & 2000 & $\begin{array}{l}\text { Controlled trials of CQI } \\
\text { and academic detailing } \\
\text { to implement a clinical } \\
\text { practice guideline for } \\
\text { depression }\end{array}$ & $\begin{array}{l}\text { Joint Commission Journal } \\
\text { on Quality Improvement } \\
\text { 26(I): 39-54 }\end{array}$ & USA & Quantitative & $\begin{array}{l}\text { Randomized } \\
\text { controlled } \\
\text { trial }\end{array}$ & Dyad & $\begin{array}{l}\text { Provider- } \\
\text { Researcher }\end{array}$ \\
\hline $\begin{array}{l}\text { Heslop, L., } \\
\text { Elsom, S., } \\
\text { Parker, N. }\end{array}$ & 2000 & $\begin{array}{l}\text { Improving continuity of } \\
\text { care across psychiatric } \\
\text { and emergency services: } \\
\text { combining patient data } \\
\text { within a participatory } \\
\text { action research } \\
\text { framework }\end{array}$ & $\begin{array}{l}\text { Journal of Advanced } \\
\text { Nursing 3I(I): 135-43 }\end{array}$ & Australia & Qualitative & $\begin{array}{l}\text { Participatory } \\
\text { action } \\
\text { research }\end{array}$ & Dyad & $\begin{array}{l}\text { Provider- } \\
\text { Researcher }\end{array}$ \\
\hline
\end{tabular}


Elliot M. Goldner et al.

\begin{tabular}{|c|c|c|c|c|c|c|c|c|}
\hline Authors & Year & Title & Publication & Country & $\begin{array}{l}\text { Research } \\
\text { approach }\end{array}$ & $\begin{array}{l}\text { Research } \\
\text { design }\end{array}$ & $\begin{array}{l}\text { Type of } \\
\text { exchange }\end{array}$ & Groups \\
\hline $\begin{array}{l}\text { Thompson, } \\
\text { C., Kinmonth, } \\
\text { A.L., Stevens, } \\
\text { L. et al. }\end{array}$ & 2000 & $\begin{array}{l}\text { Effects of a clinical } \\
\text { practice guideline and } \\
\text { practice-based education } \\
\text { on detection and } \\
\text { outcome of depression in } \\
\text { primary care: Hampshire } \\
\text { Depression Project } \\
\text { randomised controlled } \\
\text { trial }\end{array}$ & $\begin{array}{l}\text { The Lancet 355(9|99): } \\
\text { |85-9| }\end{array}$ & UK & Quantitative & $\begin{array}{l}\text { Randomized } \\
\text { controlled } \\
\text { trial }\end{array}$ & Dyad & $\begin{array}{l}\text { Provider- } \\
\text { Researcher }\end{array}$ \\
\hline $\begin{array}{l}\text { Townsend, E., } \\
\text { Birch, D.E., } \\
\text { Langley, J. } \\
\text { et al. }\end{array}$ & 2000 & $\begin{array}{l}\text { Participatory research in a } \\
\text { mental health clubhouse }\end{array}$ & $\begin{array}{l}\text { Occupational Therapy } \\
\text { Journal of Research 20(I): } \\
\text { 18-44 }\end{array}$ & USA & Qualitative & $\begin{array}{l}\text { Participatory } \\
\text { action } \\
\text { research }\end{array}$ & Dyad & $\begin{array}{l}\text { PWLE- } \\
\text { Researcher }\end{array}$ \\
\hline $\begin{array}{l}\text { Corrigan, P.W., } \\
\text { Steiner, L., } \\
\text { McCracken, } \\
\text { S.G. et al. }\end{array}$ & 2001 & $\begin{array}{l}\text { Strategies for } \\
\text { disseminating evidence- } \\
\text { based practices to staff } \\
\text { who treat people with } \\
\text { serious mental illness }\end{array}$ & $\begin{array}{l}\text { Psychiatric Services } \\
52(12): 1598-606\end{array}$ & USA & Other & Review & Dyad & $\begin{array}{l}\text { Provider- } \\
\text { Policy maker }\end{array}$ \\
\hline $\begin{array}{l}\text { Rosenheck, } \\
\text { R.A. }\end{array}$ & 2001 & $\begin{array}{l}\text { Organizational process: } \\
\text { a missing link between } \\
\text { research and practice }\end{array}$ & $\begin{array}{l}\text { Psychiatric Services 52: } \\
1607-12\end{array}$ & USA & Discussion & Discussion & Dyad & $\begin{array}{l}\text { Provider- } \\
\text { Policy maker }\end{array}$ \\
\hline $\begin{array}{l}\text { Baker, R., } \\
\text { Reddish, S., } \\
\text { Robertson, N. } \\
\text { et al. }\end{array}$ & 2001 & $\begin{array}{l}\text { Randomised controlled } \\
\text { trial of tailored strategies } \\
\text { to implement guidelines } \\
\text { for the management of } \\
\text { patients with depression } \\
\text { in general practice }\end{array}$ & $\begin{array}{l}\text { British Journal of General } \\
\text { Practice 51: 737-41 }\end{array}$ & UK & Quantitative & $\begin{array}{l}\text { Randomized } \\
\text { controlled } \\
\text { trial }\end{array}$ & Dyad & $\begin{array}{l}\text { Provider- } \\
\text { Researcher }\end{array}$ \\
\hline $\begin{array}{l}\text { Hodges, B., } \\
\text { Inch, C., } \\
\text { Silver, I. }\end{array}$ & 2001 & $\begin{array}{l}\text { Improving the psychiatric } \\
\text { knowledge, skills and } \\
\text { attitudes of primary care } \\
\text { physicians }\end{array}$ & $\begin{array}{l}\text { American Journal of } \\
\text { Psychiatry 158: 1579-86 }\end{array}$ & Canada & Other & Review & Dyad & $\begin{array}{l}\text { Provider- } \\
\text { Researcher }\end{array}$ \\
\hline $\begin{array}{l}\text { Lesage, A.D., } \\
\text { Stip, E., } \\
\text { Grunberg, F. }\end{array}$ & 2001 & $\begin{array}{l}\text { "What's up, doc?" The } \\
\text { context, limitations and } \\
\text { issues for clinicians in } \\
\text { evidence-based medicine }\end{array}$ & $\begin{array}{l}\text { Canadian Journal of } \\
\text { Psychiatry 46(5): 396-402 }\end{array}$ & Canada & Discussion & Discussion & Dyad & $\begin{array}{l}\text { Provider- } \\
\text { Researcher }\end{array}$ \\
\hline $\begin{array}{l}\text { Lin, E.H., } \\
\text { Simon, G.E., } \\
\text { Katzelnick, } \\
\text { D.J. et al. }\end{array}$ & 2001 & $\begin{array}{l}\text { Does physician } \\
\text { education on depression } \\
\text { management improve } \\
\text { treatment in primary } \\
\text { care? }\end{array}$ & $\begin{array}{l}\text { Journal of General Internal } \\
\text { Medicine 16:614-19 }\end{array}$ & USA & Quantitative & Pre-post & Dyad & $\begin{array}{l}\text { Provider- } \\
\text { Researcher }\end{array}$ \\
\hline $\begin{array}{l}\text { Naismith, S.L., } \\
\text { Hickie, I.B., } \\
\text { Scott, E.M. } \\
\text { et al. }\end{array}$ & 2001 & $\begin{array}{l}\text { Effects of mental health } \\
\text { training and clinical audit } \\
\text { on general practitioners' } \\
\text { management of common } \\
\text { mental disorders }\end{array}$ & $\begin{array}{l}\text { Medical Journal of Australia } \\
\text { I } 75 \text { (Suppl.): S42-47 }\end{array}$ & Australia & Quantitative & Pre-post & Dyad & $\begin{array}{l}\text { Provider- } \\
\text { Researcher }\end{array}$ \\
\hline
\end{tabular}


Knowledge Translation in Mental Health

\begin{tabular}{|c|c|c|c|c|c|c|c|c|}
\hline Authors & Year & Title & Publication & Country & $\begin{array}{l}\text { Research } \\
\text { approach }\end{array}$ & $\begin{array}{l}\text { Research } \\
\text { design }\end{array}$ & $\begin{array}{l}\text { Type of } \\
\text { exchange }\end{array}$ & Groups \\
\hline $\begin{array}{l}\text { van Eijk, } \\
\text { M.E.C., } \\
\text { Avorn, J., } \\
\text { Porsius, A.J. } \\
\text { et al. }\end{array}$ & 2001 & $\begin{array}{l}\text { Reducing prescribing } \\
\text { of highly anticholinergic } \\
\text { antidepressants } \\
\text { for elderly people: } \\
\text { randomised trial of } \\
\text { group versus individual } \\
\text { academic detailing } \\
\end{array}$ & $\begin{array}{l}\text { British Medical Journal } \\
\text { 322: 654-57 }\end{array}$ & Netherlands & Quantitative & $\begin{array}{l}\text { Randomized } \\
\text { controlled } \\
\text { trial }\end{array}$ & Dyad & $\begin{array}{l}\text { Provider- } \\
\text { Researcher }\end{array}$ \\
\hline $\begin{array}{l}\text { Broner, N., } \\
\text { Franczak, M., } \\
\text { Dye, C. et al. }\end{array}$ & 2001 & $\begin{array}{l}\text { Knowledge transfer, } \\
\text { policy making } \\
\text { and community } \\
\text { empowerment: a } \\
\text { consensus model } \\
\text { approach for providing } \\
\text { public mental health and } \\
\text { substance abuse services }\end{array}$ & $\begin{array}{l}\text { Psychiatric Quarterly } \\
72(1): 79-102\end{array}$ & USA & Discussion & $\begin{array}{l}\text { Discussion, } \\
\text { consensus } \\
\text { model policy } \\
\text { making }\end{array}$ & Polyad & $\begin{array}{l}\text { Provider- } \\
\text { Policy maker- } \\
\text { Researcher- } \\
\text { Other }\end{array}$ \\
\hline $\begin{array}{l}\text { Torrey, W.C., } \\
\text { Drake, R.E., } \\
\text { Dixon, L. } \\
\text { et al. }\end{array}$ & 2001 & $\begin{array}{l}\text { Implementing evidence- } \\
\text { based practices for } \\
\text { persons with severe } \\
\text { mental illnesses }\end{array}$ & $\begin{array}{l}\text { Psychiatric Services 52: } \\
45-50\end{array}$ & USA & Discussion & Discussion & Polyad & $\begin{array}{l}\text { PWLE- } \\
\text { Family- } \\
\text { Provider- } \\
\text { Policy maker- } \\
\text { Researcher }\end{array}$ \\
\hline $\begin{array}{l}\text { Bate, S.P. \& } \\
\text { Robert, G. }\end{array}$ & 2002 & $\begin{array}{l}\text { Knowledge management } \\
\text { and communities of } \\
\text { practice in the private } \\
\text { sector: lessons for } \\
\text { modernizing the National } \\
\text { Health Service in England } \\
\text { and Wales }\end{array}$ & $\begin{array}{l}\text { Public Administration } \\
80(4): 643-66\end{array}$ & UK & Discussion & Discussion & Dyad & $\begin{array}{l}\text { Provider- } \\
\text { Policy maker }\end{array}$ \\
\hline $\begin{array}{l}\text { Fleury, M.J., } \\
\text { Mercier, C., } \\
\text { Denis, J.-L. }\end{array}$ & 2002 & $\begin{array}{l}\text { Regional planning } \\
\text { implementation and its } \\
\text { impact on integration } \\
\text { of a mental healthcare } \\
\text { network }\end{array}$ & $\begin{array}{l}\text { International Journal } \\
\text { of Health Planning and } \\
\text { Management 17(4): } \\
\text { 315-32 }\end{array}$ & Canada & Qualitative & Case study & Dyad & $\begin{array}{l}\text { Provider- } \\
\text { Policy maker }\end{array}$ \\
\hline $\begin{array}{l}\text { Rempfer, M. \& } \\
\text { Knott, J. }\end{array}$ & 2002 & $\begin{array}{l}\text { Participatory action } \\
\text { research: a model for } \\
\text { establishing partnerships } \\
\text { between mental health } \\
\text { researchers and persons } \\
\text { with psychiatric disabilities }\end{array}$ & $\begin{array}{l}\text { Occupational Therapy in } \\
\text { Mental Health } 17(3 / 4) \text { : } \\
15 \mid-65\end{array}$ & USA & Discussion & $\begin{array}{l}\text { Discussion, } \\
\text { participatory } \\
\text { action theory }\end{array}$ & Dyad & $\begin{array}{l}\text { PWLE- } \\
\text { Researcher }\end{array}$ \\
\hline $\begin{array}{l}\text { Ochocka, J., } \\
\text { Janzen, R., } \\
\text { Nelson, G. }\end{array}$ & 2002 & $\begin{array}{l}\text { Sharing power and } \\
\text { knowledge: professional } \\
\text { and mental health } \\
\text { consumer/survivor } \\
\text { researchers working } \\
\text { together in a participatory } \\
\text { action research project }\end{array}$ & $\begin{array}{l}\text { Psychiatric Rehabilitation } \\
\text { Journal 25(4): } 379\end{array}$ & Canada & Qualitative & $\begin{array}{l}\text { Participatory } \\
\text { action }\end{array}$ & Polyad & $\begin{array}{l}\text { PWLE- } \\
\text { Provider- } \\
\text { Researcher }\end{array}$ \\
\hline Kates, N. & 2002 & $\begin{array}{l}\text { Nouvelles approches: } \\
\text { collaboration entre } \\
\text { dispensateurs de soins } \\
\text { primaires et de santé } \\
\text { mentale }\end{array}$ & $\begin{array}{l}\text { Santé Mentale au Québec } \\
\text { 27(2): 93-108 }\end{array}$ & Canada & Discussion & Discussion & Within group & $\begin{array}{l}\text { Provider- } \\
\text { Provider }\end{array}$ \\
\hline
\end{tabular}


Elliot M. Goldner et al.

\begin{tabular}{|c|c|c|c|c|c|c|c|c|}
\hline Authors & Year & Title & Publication & Country & $\begin{array}{l}\text { Research } \\
\text { approach }\end{array}$ & $\begin{array}{l}\text { Research } \\
\text { design }\end{array}$ & $\begin{array}{l}\text { Type of } \\
\text { exchange }\end{array}$ & Groups \\
\hline $\begin{array}{l}\text { Goering, P., } \\
\text { Butterill, D., } \\
\text { Jacobson, N. } \\
\text { et al. }\end{array}$ & 2003 & $\begin{array}{l}\text { Linkage and exchange at } \\
\text { the organizational level: } \\
\text { a model of collaboration } \\
\text { between research and } \\
\text { policy }\end{array}$ & $\begin{array}{l}\text { Journal of Health Services } \\
\text { Research \& Policy 8: } \\
14-19\end{array}$ & Canada & Discussion & Discussion & Dyad & $\begin{array}{l}\text { Policy maker- } \\
\text { Researcher }\end{array}$ \\
\hline $\begin{array}{l}\text { Azocar, F., } \\
\text { Cuffel, B., } \\
\text { Goldman, W. } \\
\text { et al. }\end{array}$ & 2003 & $\begin{array}{l}\text { The impact of evidence- } \\
\text { based guideline } \\
\text { dissemination for } \\
\text { the assessment and } \\
\text { treatment of major } \\
\text { depression in a managed } \\
\text { behavioral health care } \\
\text { organization }\end{array}$ & $\begin{array}{l}\text { Journal of Behavioral } \\
\text { Health Services Research } \\
\text { 30(1): 109-18 }\end{array}$ & USA & Quantitative & $\begin{array}{l}\text { Randomized } \\
\text { controlled } \\
\text { trial }\end{array}$ & Dyad & $\begin{array}{l}\text { Provider- } \\
\text { Researcher }\end{array}$ \\
\hline Charest, R.-M. & 2003 & $\begin{array}{l}\text { La Recherche pour servir } \\
\text { les intérêts cliniques et } \\
\text { sociaux }\end{array}$ & Psychologie Québec 5 & Canada & Discussion & Discussion & Dyad & $\begin{array}{l}\text { Provider- } \\
\text { Researcher }\end{array}$ \\
\hline $\begin{array}{l}\text { Gilbody, S.M., } \\
\text { Whitty, P.M., } \\
\text { Grimshaw, } \\
\text { J.M. et al. }\end{array}$ & 2003 & $\begin{array}{l}\text { Improving the detection } \\
\text { and management of } \\
\text { depression in primary } \\
\text { care }\end{array}$ & $\begin{array}{l}\text { Quality and Safety in } \\
\text { Health Care I2: I } 49-55\end{array}$ & UK & Discussion & $\begin{array}{l}\text { Discussion, } \\
\text { article review }\end{array}$ & Dyad & $\begin{array}{l}\text { Provider- } \\
\text { Researcher }\end{array}$ \\
\hline Lecomte, Y. & 2003 & $\begin{array}{l}\text { Développer de } \\
\text { meilleures pratiques }\end{array}$ & $\begin{array}{l}\text { Santé Mentale au Québec } \\
\text { 28(I): 9-36 }\end{array}$ & Canada & Discussion & Discussion & Dyad & $\begin{array}{l}\text { Provider- } \\
\text { Researcher }\end{array}$ \\
\hline Nadeau, L. & 2003 & $\begin{array}{l}\text { Le Processus } \\
\text { d'élaboration des lignes } \\
\text { directrices pour de } \\
\text { meilleures pratiques: } \\
\text { l'exemple des troubles } \\
\text { concomitants de santé } \\
\text { mentale, d'alcoolisme et } \\
\text { de toxicomanie }\end{array}$ & $\begin{array}{l}\text { Santé Mentale au Quebec } \\
\text { 28(I): 37-53 }\end{array}$ & Canada & Other & $\begin{array}{l}\text { Guideline } \\
\text { development }\end{array}$ & Dyad & $\begin{array}{l}\text { Provider- } \\
\text { Researcher }\end{array}$ \\
\hline Fenton, W.S. & 2003 & $\begin{array}{l}\text { Shared decision- } \\
\text { making: a model for } \\
\text { the physician-patient } \\
\text { relationship in the } 2 \text { Ist } \\
\text { century? }\end{array}$ & $\begin{array}{l}\text { Acta Psychiatrica } \\
\text { Scandinavica 107(6): } \\
401-02\end{array}$ & USA & Discussion & $\begin{array}{l}\text { Discussion, } \\
\text { shared } \\
\text { decision- } \\
\text { making }\end{array}$ & Dyad & $\begin{array}{l}\text { PWLE- } \\
\text { Provider }\end{array}$ \\
\hline $\begin{array}{l}\text { Van Korff, M., } \\
\text { Katon, W., } \\
\text { Rutter, C. } \\
\text { et al. }\end{array}$ & 2003 & $\begin{array}{l}\text { Effect on disability } \\
\text { outcomes of a } \\
\text { depression relapse } \\
\text { prevention program }\end{array}$ & $\begin{array}{l}\text { Psychosomatic Medicine } \\
65: 938-43\end{array}$ & USA & Quantitative & $\begin{array}{l}\text { Randomized } \\
\text { controlled } \\
\text { trial }\end{array}$ & Dyad & $\begin{array}{l}\text { PWLE- } \\
\text { Provider }\end{array}$ \\
\hline
\end{tabular}


Knowledge Translation in Mental Health

\begin{tabular}{|c|c|c|c|c|c|c|c|c|}
\hline Authors & Year & Title & Publication & Country & $\begin{array}{l}\text { Research } \\
\text { approach }\end{array}$ & $\begin{array}{l}\text { Research } \\
\text { design }\end{array}$ & $\begin{array}{l}\text { Type of } \\
\text { exchange }\end{array}$ & Groups \\
\hline $\begin{array}{l}\text { Ross, E., Ali, } \\
\text { A., Toner, B. }\end{array}$ & 2003 & $\begin{array}{l}\text { Investigating issues } \\
\text { surrounding depression } \\
\text { in adolescent girls across } \\
\text { Ontario: a participatory } \\
\text { action research project }\end{array}$ & $\begin{array}{l}\text { Canadian Journal of } \\
\text { Community Mental Health } \\
\text { 22(I): 55-68 }\end{array}$ & Canada & Qualitative & $\begin{array}{l}\text { Participatory } \\
\text { action }\end{array}$ & Dyad & $\begin{array}{l}\text { PWLE- } \\
\text { Researcher }\end{array}$ \\
\hline Ganju, V. & 2003 & $\begin{array}{l}\text { Implementation of } \\
\text { evidence-based practices } \\
\text { in state mental health } \\
\text { systems }\end{array}$ & $\begin{array}{l}\text { Schizophrenia Bulletin } \\
29(1):|25-3|\end{array}$ & USA & Discussion & Discussion & Polyad & $\begin{array}{l}\text { Provider- } \\
\text { Policy maker- } \\
\text { Researcher }\end{array}$ \\
\hline $\begin{array}{l}\text { Malm, U., } \\
\text { Ivarsson, B., } \\
\text { Allebeck, P. } \\
\text { et al. }\end{array}$ & 2003 & $\begin{array}{l}\text { Integrated care in } \\
\text { schizophrenia: a two- } \\
\text { year randomized } \\
\text { controlled study of } \\
\text { two community-based } \\
\text { treatment programs }\end{array}$ & $\begin{array}{l}\text { Acta Psychiatrica } \\
\text { Scandinavica 107(6): } \\
4 \mid 5-23\end{array}$ & Sweden & Quantitative & $\begin{array}{l}\text { Randomized } \\
\text { controlled } \\
\text { trial }\end{array}$ & Polyad & $\begin{array}{l}\text { PWLE- } \\
\text { Family- } \\
\text { Provider }\end{array}$ \\
\hline $\begin{array}{l}\text { Kirmayer, L., } \\
\text { Simpson, C., } \\
\text { Cargo, M. }\end{array}$ & 2003 & $\begin{array}{l}\text { Healing traditions: } \\
\text { culture, community and } \\
\text { mental health promotion } \\
\text { with Canadian Aboriginal } \\
\text { peoples }\end{array}$ & $\begin{array}{l}\text { Australasian Psychiatry } \\
\text { I I(sI): SI 5-S23 }\end{array}$ & Canada & Discussion & Discussion & Polyad & $\begin{array}{l}\text { PWLE- } \\
\text { Family- } \\
\text { Provider- } \\
\text { Policy maker- } \\
\text { Researcher- } \\
\text { Other }\end{array}$ \\
\hline $\begin{array}{l}\text { Vingilis, E., } \\
\text { Hartford, K., } \\
\text { Schrecker, T. } \\
\text { et al. }\end{array}$ & 2003 & $\begin{array}{l}\text { Integrating knowledge } \\
\text { generation with } \\
\text { knowledge diffusion } \\
\text { and utilization: a case } \\
\text { study analysis of the } \\
\text { consortium for applied } \\
\text { research and evaluation } \\
\text { in mental health. }\end{array}$ & $\begin{array}{l}\text { Canadian Journal of Public } \\
\text { Health 94(6): 468-7| }\end{array}$ & Canada & Qualitative & $\begin{array}{l}\text { Participatory } \\
\text { action, single } \\
\text { case design }\end{array}$ & Polyad & $\begin{array}{l}\text { PWLE- } \\
\text { Family- } \\
\text { Provider- } \\
\text { Policy maker- } \\
\text { Researcher- } \\
\text { Other }\end{array}$ \\
\hline $\begin{array}{l}\text { Sullivan, M.P., } \\
\text { Kessler, L., } \\
\text { Le Clair, J.K. } \\
\text { et al. }\end{array}$ & 2004 & $\begin{array}{l}\text { Defining best practices } \\
\text { for specialty geriatric } \\
\text { mental health outreach } \\
\text { services: lessons for } \\
\text { implementing mental } \\
\text { health reform }\end{array}$ & $\begin{array}{l}\text { Canadian Journal of } \\
\text { Psychiatry 49(7): 458-66 }\end{array}$ & Canada & Other & Review & Dyad & $\begin{array}{l}\text { Provider- } \\
\text { Policy maker }\end{array}$ \\
\hline $\begin{array}{l}\text { Van Os, J., } \\
\text { Altamura, } \\
\text { A.C., Bobes, J. } \\
\text { et al. }\end{array}$ & 2004 & $\begin{array}{l}\text { Evaluation of the two- } \\
\text { way communication } \\
\text { checklist as a clinical } \\
\text { intervention: results } \\
\text { of a multinational, } \\
\text { randomized controlled } \\
\text { trial }\end{array}$ & $\begin{array}{l}\text { British Journal of Psychiatry } \\
\text { 184: 79-83 }\end{array}$ & Netherlands & Quantitative & $\begin{array}{l}\text { Questionnaire } \\
\text { data }\end{array}$ & Dyad & $\begin{array}{l}\text { PWLE- } \\
\text { Provider }\end{array}$ \\
\hline Neufeldt, A.H. & 2004 & $\begin{array}{l}\text { What does it take } \\
\text { to transform mental } \\
\text { health knowledge into } \\
\text { workplace practice? } \\
\text { Towards a theory of } \\
\text { action }\end{array}$ & $\begin{array}{l}\text { HealthcarePapers 5(2): } \\
\text { 1 18-32 }\end{array}$ & Canada & Discussion & Discussion & Polyad & $\begin{array}{l}\text { Provider- } \\
\text { Policy maker- } \\
\text { Researcher }\end{array}$ \\
\hline Gotham, H.J. & 2004 & $\begin{array}{l}\text { Diffusion of mental } \\
\text { health and substance } \\
\text { abuse treatments: } \\
\text { development, } \\
\text { dissemination and } \\
\text { implementation }\end{array}$ & $\begin{array}{l}\text { Clinical Psychology } \\
\text { Science and Practice } \\
\text { I I (2): 160-77 }\end{array}$ & USA & Discussion & Discussion & Polyad & $\begin{array}{l}\text { Provider- } \\
\text { Policy maker- } \\
\text { Researcher }\end{array}$ \\
\hline
\end{tabular}


Elliot M. Goldner et al.

\begin{tabular}{|c|c|c|c|c|c|c|c|c|}
\hline Authors & Year & Title & Publication & Country & $\begin{array}{l}\text { Research } \\
\text { approach }\end{array}$ & $\begin{array}{l}\text { Research } \\
\text { design }\end{array}$ & $\begin{array}{l}\text { Type of } \\
\text { exchange }\end{array}$ & Groups \\
\hline $\begin{array}{l}\text { de Crespigny, } \\
\text { C., Emden, } \\
\text { C., Kowanko, } \\
\text { I., Murray, H. }\end{array}$ & 2004 & $\begin{array}{l}\text { A partnership model } \\
\text { for ethical Indigenous } \\
\text { research }\end{array}$ & $\begin{array}{l}\text { Collegian: Journal of the } \\
\text { Royal College of Nursing } \\
\text { Australia I I (4): 7-13 }\end{array}$ & Australia & Qualitative & $\begin{array}{l}\text { Participatory } \\
\text { action } \\
\text { research }\end{array}$ & Polyad & $\begin{array}{l}\text { PWLE- } \\
\text { Family- } \\
\text { Provider- } \\
\text { Researcher }\end{array}$ \\
\hline $\begin{array}{l}\text { Sadavoy, J., } \\
\text { Meier, R., } \\
\text { Ong, A.Y. }\end{array}$ & 2004 & $\begin{array}{l}\text { Barriers to access to } \\
\text { mental health services } \\
\text { for ethnic seniors: the } \\
\text { Toronto Study }\end{array}$ & $\begin{array}{l}\text { Canadian Journal of } \\
\text { Psychiatry 49(3): 192-99 }\end{array}$ & Canada & Qualitative & $\begin{array}{l}\text { Participatory } \\
\text { action, } \\
\text { grounded } \\
\text { theory }\end{array}$ & Polyad & $\begin{array}{l}\text { PWLE- } \\
\text { Family- } \\
\text { Provider- } \\
\text { Researcher }\end{array}$ \\
\hline $\begin{array}{l}\text { Minore, B., } \\
\text { Boone, M., } \\
\text { Katt, M. et al. }\end{array}$ & 2004 & $\begin{array}{l}\text { Addressing the realities } \\
\text { of healthcare in northern } \\
\text { Aboriginal communities } \\
\text { through participatory } \\
\text { action research }\end{array}$ & $\begin{array}{l}\text { Journal of Interprofessional } \\
\text { Care I8(4): 360-68 }\end{array}$ & Canada & Other & $\begin{array}{l}\text { Mixed } \\
\text { methods, } \\
\text { participatory } \\
\text { action } \\
\text { research }\end{array}$ & Polyad & $\begin{array}{l}\text { PWLE- } \\
\text { Provider- } \\
\text { Policy maker }\end{array}$ \\
\hline $\begin{array}{l}\text { Sundar, P. \& } \\
\text { Ochocka, J. }\end{array}$ & 2004 & $\begin{array}{l}\text { Bridging the gap between } \\
\text { dreams and realities } \\
\text { related to employment } \\
\text { and mental health: } \\
\text { implications for policy } \\
\text { and practice }\end{array}$ & $\begin{array}{l}\text { Canadian Journal of } \\
\text { Community Mental Health } \\
\text { 23(I): 75-89 }\end{array}$ & Canada & Qualitative & $\begin{array}{l}\text { Participatory } \\
\text { action }\end{array}$ & Polyad & $\begin{array}{l}\text { PWLE- } \\
\text { Provider- } \\
\text { Researcher }\end{array}$ \\
\hline $\begin{array}{l}\text { Waddell, C., } \\
\text { Lavis, J.N., } \\
\text { Abelson, J. } \\
\text { et al. }\end{array}$ & 2005 & $\begin{array}{l}\text { Research use in children's } \\
\text { mental health policy in } \\
\text { Canada: maintaining } \\
\text { vigilance amid ambiguity }\end{array}$ & $\begin{array}{l}\text { Social Science \& Medicine } \\
6 \text { (8): 1649-57 }\end{array}$ & Canada & Qualitative & Interviews & Dyad & $\begin{array}{l}\text { Policy maker- } \\
\text { Researcher }\end{array}$ \\
\hline $\begin{array}{l}\text { Bourbonnais, } \\
\text { R., Gauthier, } \\
\text { N., Vézina, M. } \\
\text { et al. }\end{array}$ & 2005 & $\begin{array}{l}\text { Une intervention en } \\
\text { centres d'hébergement } \\
\text { et de soins de longue } \\
\text { durée visant à réduire } \\
\text { les problèmes de santé } \\
\text { mentale liés au travail }\end{array}$ & Pistes 7(2) & Canada & Other & $\begin{array}{l}\text { Mixed } \\
\text { methods, } \\
\text { participatory } \\
\text { action } \\
\text { research, } \\
\text { qualitative } \\
\text { evaluation, } \\
\text { questionnaires }\end{array}$ & Dyad & $\begin{array}{l}\text { Provider- } \\
\text { Researcher }\end{array}$ \\
\hline $\begin{array}{l}\text { Eisses, A.M.H. } \\
\text { et al. }\end{array}$ & 2005 & $\begin{array}{l}\text { Care staff training in } \\
\text { detection of depression } \\
\text { in residential homes for } \\
\text { the elderly }\end{array}$ & $\begin{array}{l}\text { British Journal of Psychiatry } \\
\text { 186: 404-09 }\end{array}$ & Netherlands & Quantitative & $\begin{array}{l}\text { Randomized } \\
\text { controlled } \\
\text { trial }\end{array}$ & Dyad & $\begin{array}{l}\text { Provider- } \\
\text { Researcher }\end{array}$ \\
\hline $\begin{array}{l}\text { Rousseau, } \\
\text { C., Alain, N., } \\
\text { DePlaen, S. } \\
\text { et al. }\end{array}$ & 2005 & $\begin{array}{l}\text { Repenser la formation } \\
\text { continue dans le } \\
\text { réseau de la santé } \\
\text { et des services } \\
\text { sociaux: l'expérience } \\
\text { des séminaires } \\
\text { interinstitutionnels } \\
\text { en intervention } \\
\text { transculturelle }\end{array}$ & $\begin{array}{l}\text { Nouvelles Pratiques } \\
\text { Sociales 17(2): 109-25 }\end{array}$ & Canada & Qualitative & Case study & Dyad & $\begin{array}{l}\text { Provider- } \\
\text { Researcher }\end{array}$ \\
\hline
\end{tabular}


Knowledge Translation in Mental Health

\begin{tabular}{|c|c|c|c|c|c|c|c|c|}
\hline Authors & Year & Title & Publication & Country & $\begin{array}{l}\text { Research } \\
\text { approach }\end{array}$ & $\begin{array}{l}\text { Research } \\
\text { design }\end{array}$ & $\begin{array}{l}\text { Type of } \\
\text { exchange }\end{array}$ & Groups \\
\hline $\begin{array}{l}\text { Ruzek, J.l., } \\
\text { Friedman, } \\
\text { M.J., Murray, } \\
\text { S. }\end{array}$ & 2005 & $\begin{array}{l}\text { Toward a knowledge } \\
\text { management system } \\
\text { for postraumatic stress } \\
\text { disorder treatment in } \\
\text { veterans' healthcare }\end{array}$ & $\begin{array}{l}\text { Psychiatric Annals 35(I I): } \\
911-20\end{array}$ & USA & Discussion & Discussion & Dyad & $\begin{array}{l}\text { Provider- } \\
\text { Researcher }\end{array}$ \\
\hline $\begin{array}{l}\text { Bowen, S. \& } \\
\text { Martens, P. }\end{array}$ & 2005 & $\begin{array}{l}\text { Demystifying knowledge } \\
\text { translation: learning from } \\
\text { the community }\end{array}$ & $\begin{array}{l}\text { Journal of Health Services } \\
\text { Research \& Policy 10: } \\
\text { 203-11 }\end{array}$ & Canada & Qualitative & $\begin{array}{l}\text { Participatory } \\
\text { action } \\
\text { research }\end{array}$ & Dyad & $\begin{array}{l}\text { Provider- } \\
\text { Researcher }\end{array}$ \\
\hline $\begin{array}{l}\text { Glisson, C. \& } \\
\text { Schoenwald, } \\
\text { S.K. }\end{array}$ & 2005 & $\begin{array}{l}\text { The ARC organizational } \\
\text { and community } \\
\text { intervention strategy for } \\
\text { implementing evidence- } \\
\text { based children's mental } \\
\text { health treatments }\end{array}$ & $\begin{array}{l}\text { Mental Health Services } \\
\text { Research 7(4): 243-59 }\end{array}$ & USA & Other & Review & Polyad & $\begin{array}{l}\text { Family- } \\
\text { Provider- } \\
\text { Policy maker- } \\
\text { Other }\end{array}$ \\
\hline $\begin{array}{l}\text { Jorm, A.F., } \\
\text { Christensen, } \\
\text { H., Griffiths, } \\
\text { K.M. }\end{array}$ & 2005 & $\begin{array}{l}\text { The impact of } \\
\text { beyondblue: the national } \\
\text { depression initiative on } \\
\text { the Australian public's } \\
\text { recognition of depression } \\
\text { and beliefs about } \\
\text { treatments }\end{array}$ & $\begin{array}{l}\text { Australian \& New Zealand } \\
\text { Journal of Psychiatry 39(4): } \\
\text { 248-54 }\end{array}$ & Australia & Quantitative & $\begin{array}{l}\text { Non- } \\
\text { randomized } \\
\text { controlled } \\
\text { trial }\end{array}$ & Polyad & $\begin{array}{l}\text { Policy maker- } \\
\text { Researcher- } \\
\text { Other }\end{array}$ \\
\hline Spaniol, S. & 2005 & $\begin{array}{l}\text { "Learned hopefulness": } \\
\text { an arts-based approach } \\
\text { to participatory action } \\
\text { research }\end{array}$ & Art Therapy 22(2): 86-91 & USA & Qualitative & $\begin{array}{l}\text { Participatory } \\
\text { action, } \\
\text { arts-based, } \\
\text { conference } \\
\text { format }\end{array}$ & Polyad & $\begin{array}{l}\text { PWLE- } \\
\text { Provider- } \\
\text { Researcher }\end{array}$ \\
\hline $\begin{array}{l}\text { Perreault, M., } \\
\text { Bonin, J.-P., } \\
\text { Veilleux, R. } \\
\text { et al. }\end{array}$ & 2005 & $\begin{array}{l}\text { Expérience de formation } \\
\text { croisée dans un contexte } \\
\text { d'intégration des services } \\
\text { en réseau dans le sud- } \\
\text { ouest de Montréal }\end{array}$ & $\begin{array}{l}\text { Canadian Journal of } \\
\text { Community Mental Health } \\
\text { 24(I): 35-49 }\end{array}$ & Canada & Other & $\begin{array}{l}\text { Mixed } \\
\text { methods, } \\
\text { questionnaires } \\
\text { and focus } \\
\text { groups }\end{array}$ & Within group & $\begin{array}{l}\text { Provider- } \\
\text { Provider }\end{array}$ \\
\hline $\begin{array}{l}\text { Garland, A.F., } \\
\text { Plemmons, } \\
\text { D., Koontz, L. }\end{array}$ & 2006 & $\begin{array}{l}\text { Research-practice } \\
\text { partnership in mental } \\
\text { health: lessons from } \\
\text { participants }\end{array}$ & $\begin{array}{l}\text { Administration and Policy } \\
\text { in Mental Health and } \\
\text { Mental Health Services } \\
\text { Research 33(5): 517-28 }\end{array}$ & USA & Qualitative & $\begin{array}{l}\text { Semi- } \\
\text { structured } \\
\text { interviewing }\end{array}$ & Dyad & $\begin{array}{l}\text { Provider- } \\
\text { Researcher }\end{array}$ \\
\hline $\begin{array}{l}\text { Hahn, S., } \\
\text { Needham, l., } \\
\text { Abderhalden, } \\
\text { C. }\end{array}$ & 2006 & $\begin{array}{l}\text { The effect of a training } \\
\text { course on mental health } \\
\text { nurses' attitudes on } \\
\text { the reasons of patient } \\
\text { aggression and its } \\
\text { management }\end{array}$ & $\begin{array}{l}\text { Journal of Psychiatric and } \\
\text { Mental Health Nursing } \\
\text { I2(2): 197-204 }\end{array}$ & Switzerland & Quantitative & $\begin{array}{l}\text { Quasi- } \\
\text { experimental, } \\
\text { pre-post test }\end{array}$ & Dyad & $\begin{array}{l}\text { Provider- } \\
\text { Researcher }\end{array}$ \\
\hline $\begin{array}{l}\text { Horvitz- } \\
\text { Lennon, M., } \\
\text { Kilbourne, } \\
\text { A.M., Pincus, } \\
\text { H.A. }\end{array}$ & 2006 & $\begin{array}{l}\text { From silos to bridges: } \\
\text { meeting the general } \\
\text { healthcare needs of } \\
\text { adults with severe mental } \\
\text { illnesses }\end{array}$ & $\begin{array}{l}\text { Health Affairs 25(3): } \\
659-69\end{array}$ & USA & Discussion & Discussion & Dyad & $\begin{array}{l}\text { Provider- } \\
\text { Researcher }\end{array}$ \\
\hline
\end{tabular}


Elliot M. Goldner et al.

\begin{tabular}{|c|c|c|c|c|c|c|c|c|}
\hline Authors & Year & Title & Publication & Country & $\begin{array}{l}\text { Research } \\
\text { approach }\end{array}$ & $\begin{array}{l}\text { Research } \\
\text { design }\end{array}$ & $\begin{array}{l}\text { Type of } \\
\text { exchange }\end{array}$ & Groups \\
\hline $\begin{array}{l}\text { Ingadottir, E. \& } \\
\text { Thome, M. }\end{array}$ & 2006 & $\begin{array}{l}\text { Evaluation of a web- } \\
\text { based course for } \\
\text { community nurses on } \\
\text { postpartum emotional } \\
\text { distress }\end{array}$ & $\begin{array}{l}\text { Scandinavian Journal } \\
\text { of Caring Sciences 20: } \\
86-92\end{array}$ & Iceland & Quantitative & $\begin{array}{l}\text { Non- } \\
\text { randomized } \\
\text { controlled } \\
\text { trial }\end{array}$ & Dyad & $\begin{array}{l}\text { Provider- } \\
\text { Researcher }\end{array}$ \\
\hline $\begin{array}{l}\text { Rinfret- } \\
\text { Raynor, M., } \\
\text { Dubé, M., } \\
\text { Drouin, C. }\end{array}$ & 2006 & $\begin{array}{l}\text { Le Dépistage de la } \\
\text { violence conjugale dans } \\
\text { les centre hospitaliers: } \\
\text { implantation et évaluation } \\
\text { d'un ensemble d'outils }\end{array}$ & $\begin{array}{l}\text { Nouvelles Pratiques } \\
\text { Sociales 19(1): 72-90 }\end{array}$ & Canada & Qualitative & Case study & Dyad & $\begin{array}{l}\text { Provider- } \\
\text { Researcher }\end{array}$ \\
\hline $\begin{array}{l}\text { Wimpenny, } \\
\text { K., Forsyth, } \\
\text { K., Jones, C., } \\
\text { Evans, E., } \\
\text { Colley, J. }\end{array}$ & 2006 & $\begin{array}{l}\text { Group reflective } \\
\text { supervision: thinking } \\
\text { with theory to develop } \\
\text { practice }\end{array}$ & $\begin{array}{l}\text { British Journal of } \\
\text { Occupational Therapy } \\
69(9): 423-28\end{array}$ & UK & Qualitative & $\begin{array}{l}\text { Participatory } \\
\text { action } \\
\text { research }\end{array}$ & Dyad & $\begin{array}{l}\text { Provider- } \\
\text { Researcher }\end{array}$ \\
\hline $\begin{array}{l}\text { Beresford, P. \& } \\
\text { Branfield, F. }\end{array}$ & 2006 & $\begin{array}{l}\text { Developing inclusive } \\
\text { partnerships: user-defined } \\
\text { outcomes, networking } \\
\text { and knowledge - a case } \\
\text { study }\end{array}$ & $\begin{array}{l}\text { Health and Social Care } \\
\text { in the Community I } 4(5) \text { : } \\
436-44\end{array}$ & UK & Qualitative & Interviews & Dyad & $\begin{array}{l}\text { PWLE- } \\
\text { Provider }\end{array}$ \\
\hline $\begin{array}{l}\text { Deegan, P.E. \& } \\
\text { Drake, R.E. }\end{array}$ & 2006 & $\begin{array}{l}\text { Shared decision- } \\
\text { making and medication } \\
\text { management in the } \\
\text { recovery process }\end{array}$ & $\begin{array}{l}\text { Psychiatric Services } \\
57(11): 1636-39\end{array}$ & USA & Discussion & Discussion & Dyad & $\begin{array}{l}\text { PWLE- } \\
\text { Provider }\end{array}$ \\
\hline $\begin{array}{l}\text { Hamann, J., } \\
\text { Langer, B., } \\
\text { Winkler, V. } \\
\text { et al. }\end{array}$ & 2006 & $\begin{array}{l}\text { Shared decision-making } \\
\text { for inpatients with } \\
\text { schizophrenia }\end{array}$ & $\begin{array}{l}\text { Acta Psychiatrica } \\
\text { Scandinavica | | 4: 265-73 }\end{array}$ & Germany & Quantitative & $\begin{array}{l}\text { Randomized } \\
\text { controlled } \\
\text { trial }\end{array}$ & Dyad & $\begin{array}{l}\text { PWLE- } \\
\text { Provider }\end{array}$ \\
\hline $\begin{array}{l}\text { Jared, R., } \\
\text { Adams, J.R., } \\
\text { Drake, R.E. }\end{array}$ & 2006 & $\begin{array}{l}\text { Shared decision-making } \\
\text { and evidence-based } \\
\text { practice }\end{array}$ & $\begin{array}{l}\text { Community Mental Health } \\
\text { Journal 42(I): 87-105 }\end{array}$ & USA & Discussion & Discussion & Dyad & $\begin{array}{l}\text { PWLE- } \\
\text { Provider }\end{array}$ \\
\hline Mahone, I.H. & 2006 & $\begin{array}{l}\text { Medication decision- } \\
\text { making by persons with } \\
\text { serious mental illness }\end{array}$ & $\begin{array}{l}\text { Dissertation Abstracts } \\
\text { International: Section } \\
\text { B: The Sciences and } \\
\text { Engineering. US: } \\
\text { ProQuest Information \& } \\
\text { Learning }\end{array}$ & USA & Quantitative & $\begin{array}{l}\text { Cross- } \\
\text { sectional } \\
\text { correlational } \\
\text { study }\end{array}$ & Dyad & $\begin{array}{l}\text { PWLE- } \\
\text { Provider }\end{array}$ \\
\hline $\begin{array}{l}\text { Penn, D.L., } \\
\text { Simpson, } \\
\text { L.E., Leggett, } \\
\text { S., Edie, G., } \\
\text { Wood, L. }\end{array}$ & 2006 & $\begin{array}{l}\text { The development of } \\
\text { a website to promote } \\
\text { the mental and physical } \\
\text { health of sons and } \\
\text { daughters of Vietnam } \\
\text { veterans of Australia }\end{array}$ & $\begin{array}{l}\text { Journal of Consumer } \\
\text { Health on the Internet } \\
\text { IO(4): 45-63 }\end{array}$ & Australia & Qualitative & $\begin{array}{l}\text { Participatory } \\
\text { action }\end{array}$ & Dyad & $\begin{array}{l}\text { PWLE- } \\
\text { Researcher }\end{array}$ \\
\hline
\end{tabular}


Knowledge Translation in Mental Health

\begin{tabular}{|c|c|c|c|c|c|c|c|c|}
\hline Authors & Year & Title & Publication & Country & $\begin{array}{l}\text { Research } \\
\text { approach }\end{array}$ & $\begin{array}{l}\text { Research } \\
\text { design }\end{array}$ & $\begin{array}{l}\text { Type of } \\
\text { exchange }\end{array}$ & Groups \\
\hline $\begin{array}{l}\text { Ditrano, C.J., } \\
\text { Silverstein, } \\
\text { L.B., } \\
\text { Bordeaux, L. }\end{array}$ & 2006 & $\begin{array}{l}\text { Listening to parents' } \\
\text { voices: participatory } \\
\text { action research in the } \\
\text { schools }\end{array}$ & $\begin{array}{l}\text { Professional Psychology: } \\
\text { Research and Practice 37: } \\
\text { 359-66 }\end{array}$ & USA & Qualitative & $\begin{array}{l}\text { Participatory } \\
\text { action } \\
\text { research }\end{array}$ & Polyad & $\begin{array}{l}\text { Family- } \\
\text { Provider- } \\
\text { Researcher }\end{array}$ \\
\hline $\begin{array}{l}\text { Knapp, M., } \\
\text { Funk, M., } \\
\text { Curran, C. } \\
\text { et al. }\end{array}$ & 2006 & $\begin{array}{l}\text { Economic barriers to } \\
\text { better mental health } \\
\text { practice and policy }\end{array}$ & $\begin{array}{l}\text { Health Policy and Planning } \\
21(3): 157-70\end{array}$ & UK & Discussion & Discussion & Polyad & $\begin{array}{l}\text { Provider- } \\
\text { Policy maker- } \\
\text { Other }\end{array}$ \\
\hline $\begin{array}{l}\text { Knightbridge, } \\
\text { S.M., King, R., } \\
\text { Rolfe, T.J. }\end{array}$ & 2006 & $\begin{array}{l}\text { Using participatory action } \\
\text { research in a community- } \\
\text { based initiative addressing } \\
\text { complex mental health } \\
\text { needs }\end{array}$ & $\begin{array}{l}\text { Australian \& New Zealand } \\
\text { Journal of Psychiatry 40(4): } \\
325-32\end{array}$ & Australia & Qualitative & $\begin{array}{l}\text { Participatory } \\
\text { action } \\
\text { research }\end{array}$ & Polyad & $\begin{array}{l}\text { PWLE- } \\
\text { Family- } \\
\text { Provider- } \\
\text { Policy maker- } \\
\text { Researcher }\end{array}$ \\
\hline $\begin{array}{l}\text { Bluthenthal, } \\
\text { R.N., Jones, } \\
\text { L., Fackler- } \\
\text { Lowrie, N. }\end{array}$ & 2006 & $\begin{array}{l}\text { Witness for wellness: } \\
\text { preliminary findings from } \\
\text { a community-academic } \\
\text { participatory research } \\
\text { initiative }\end{array}$ & $\begin{array}{l}\text { Ethnicity and Disease } \\
\text { 16(s): } 18-34\end{array}$ & USA & Other & $\begin{array}{l}\text { Mixed } \\
\text { methods, } \\
\text { participatory } \\
\text { action } \\
\text { research }\end{array}$ & Polyad & $\begin{array}{l}\text { PWLE- } \\
\text { Family- } \\
\text { Provider- } \\
\text { Researcher- } \\
\text { Other }\end{array}$ \\
\hline $\begin{array}{l}\text { Thomas, P., } \\
\text { Seebohm, P., } \\
\text { Henderson, } \\
\text { P., Munn- } \\
\text { Giddings, C., } \\
\text { Yasmeen, S. }\end{array}$ & 2006 & $\begin{array}{l}\text { Tackling race } \\
\text { inequalities: community } \\
\text { development, mental } \\
\text { health and diversity }\end{array}$ & $\begin{array}{l}\text { Journal of Public Mental } \\
\text { Health 5(2): 13-19 }\end{array}$ & Canada & Qualitative & Focus groups & Polyad & $\begin{array}{l}\text { PWLE-Policy } \\
\text { maker- } \\
\text { Researcher }\end{array}$ \\
\hline $\begin{array}{l}\text { Craven, M.A. } \\
\text { \& Bland, R. }\end{array}$ & 2006 & $\begin{array}{l}\text { Better practices in } \\
\text { collaborative mental } \\
\text { healthcare: an analysis of } \\
\text { the evidence base }\end{array}$ & $\begin{array}{l}\text { Canadian Journal of } \\
\text { Psychiatry 5I(SI): } 7 \text { S-74S }\end{array}$ & Canada & Other & $\begin{array}{l}\text { Systematic } \\
\text { review }\end{array}$ & Polyad & $\begin{array}{l}\text { PWLE- } \\
\text { Provider- } \\
\text { Decision- } \\
\text { makers }\end{array}$ \\
\hline $\begin{array}{l}\text { Wills, C.E. } \\
\text { \& Holmes- } \\
\text { Rovne, M. }\end{array}$ & 2006 & $\begin{array}{l}\text { Integrating decision- } \\
\text { making and mental health } \\
\text { interventions research: } \\
\text { research directions }\end{array}$ & $\begin{array}{l}\text { Clinical Psychology: } \\
\text { Science and Practice } \\
\text { 13(I): 9-25 }\end{array}$ & USA & Discussion & Discussion & Polyad & $\begin{array}{l}\text { PWLE- } \\
\text { Provider- } \\
\text { Policy maker }\end{array}$ \\
\hline $\begin{array}{l}\text { Waddell, C., } \\
\text { Shepherd, } \\
\text { C.A., Lavis, } \\
\text { J.N. et al. }\end{array}$ & 2007 & $\begin{array}{l}\text { Balancing rigour and } \\
\text { relevance: researchers' } \\
\text { contributions to children's } \\
\text { mental health policy in } \\
\text { Canada }\end{array}$ & $\begin{array}{l}\text { Evidence \& Policy: A } \\
\text { Journal of Research, } \\
\text { Debate and Practice 3(2): } \\
\text { |8|-95 }\end{array}$ & Canada & Qualitative & Interviews & Dyad & $\begin{array}{l}\text { Policy maker- } \\
\text { Researcher }\end{array}$ \\
\hline Lesage, A.D. & 2007 & $\begin{array}{l}\text { Les Programmes de } \\
\text { premier épisode de } \\
\text { schizophrénie et une } \\
\text { médecine fondée sur } \\
\text { les données factuelles : } \\
\text { un cas de syndrome des } \\
\text { habits de l'empereur? }\end{array}$ & $\begin{array}{l}\text { Canadian Journal of } \\
\text { Psychiatry 32(I): 333-49 }\end{array}$ & Canada & Discussion & Discussion & Dyad & $\begin{array}{l}\text { Provider- } \\
\text { Researcher }\end{array}$ \\
\hline
\end{tabular}


Elliot M. Goldner et al.

\begin{tabular}{|c|c|c|c|c|c|c|c|c|}
\hline Authors & Year & Title & Publication & Country & $\begin{array}{l}\text { Research } \\
\text { approach }\end{array}$ & $\begin{array}{l}\text { Research } \\
\text { design }\end{array}$ & $\begin{array}{l}\text { Type of } \\
\text { exchange }\end{array}$ & Groups \\
\hline $\begin{array}{l}\text { Wong, S.Y.S., } \\
\text { Cheung, } \\
\text { A.K.Y., Lee, A. } \\
\text { et al. }\end{array}$ & 2007 & $\begin{array}{l}\text { Improving general } \\
\text { practitioners' interviewing } \\
\text { skills in managing patients } \\
\text { with depression and } \\
\text { anxiety: a randomized } \\
\text { controlled clinical trial }\end{array}$ & $\begin{array}{l}\text { Medical Teacher 29: } \\
\text { el75-el } 83\end{array}$ & China & Quantitative & $\begin{array}{l}\text { Randomized } \\
\text { controlled } \\
\text { trial }\end{array}$ & Dyad & $\begin{array}{l}\text { Provider- } \\
\text { Researcher }\end{array}$ \\
\hline $\begin{array}{l}\text { Brekke, } \\
\text { J.S., Ell, K., } \\
\text { Palinkas, L. }\end{array}$ & 2007 & $\begin{array}{l}\text { Translational science at } \\
\text { the National Institute of } \\
\text { Mental Health: can social } \\
\text { work take its rightful } \\
\text { place? }\end{array}$ & $\begin{array}{l}\text { Research on Social Work } \\
\text { Practice I7(I): 123-33 }\end{array}$ & USA & Discussion & Discussion & Dyad & $\begin{array}{l}\text { Provider- } \\
\text { Researcher }\end{array}$ \\
\hline $\begin{array}{l}\text { Adams, J.R., } \\
\text { Drake, R.E., } \\
\text { Wolford, G.L. }\end{array}$ & 2007 & $\begin{array}{l}\text { Shared decision-making } \\
\text { preferences of people } \\
\text { with severe mental illness }\end{array}$ & $\begin{array}{l}\text { Psychiatric Services 58: } \\
|2| 9-2 \mid\end{array}$ & USA & Quantitative & $\begin{array}{l}\text { Cross- } \\
\text { sectional } \\
\text { survey }\end{array}$ & Dyad & $\begin{array}{l}\text { PWLE- } \\
\text { Provider }\end{array}$ \\
\hline Deegan, P.E. & 2007 & $\begin{array}{l}\text { The lived experience } \\
\text { of using psychiatric } \\
\text { medication in the } \\
\text { recovery process and a } \\
\text { shared decision-making } \\
\text { program to support in } \\
\text { the recovery process }\end{array}$ & $\begin{array}{l}\text { Psychiatric Rehabilitation } \\
\text { Journal 31(1): 62-69 }\end{array}$ & USA & Discussion & Discussion & Dyad & $\begin{array}{l}\text { PWLE- } \\
\text { Provider }\end{array}$ \\
\hline $\begin{array}{l}\text { Loh, A., } \\
\text { Simon, D., } \\
\text { Wills, C.E. } \\
\text { et al. }\end{array}$ & 2007 & $\begin{array}{l}\text { The effects of a shared } \\
\text { decision-making } \\
\text { intervention in primary } \\
\text { care of depression: a } \\
\text { cluster-randomized } \\
\text { controlled trial }\end{array}$ & $\begin{array}{l}\text { Patient Education and } \\
\text { Counseling 67(3): 324-32 }\end{array}$ & Germany & Quantitative & $\begin{array}{l}\text { Cluster- } \\
\text { randomized } \\
\text { controlled } \\
\text { trial }\end{array}$ & Dyad & $\begin{array}{l}\text { PWLE- } \\
\text { Provider }\end{array}$ \\
\hline $\begin{array}{l}\text { Priebe, S., } \\
\text { McCabe, R., } \\
\text { Bullenkamp, J. } \\
\text { et al. }\end{array}$ & 2007 & $\begin{array}{l}\text { Structured patient- } \\
\text { clinician communication } \\
\text { and one-year outcome } \\
\text { in community mental } \\
\text { healthcare }\end{array}$ & $\begin{array}{l}\text { British Journal of Psychiatry } \\
\text { 191: 420-26 }\end{array}$ & UK & Quantitative & $\begin{array}{l}\text { Cluster- } \\
\text { randomized } \\
\text { controlled } \\
\text { trial }\end{array}$ & Dyad & $\begin{array}{l}\text { PWLE- } \\
\text { Provider }\end{array}$ \\
\hline $\begin{array}{l}\text { Schauer, C., } \\
\text { Everett, A., } \\
\text { del Vecchio, P. } \\
\text { et al. }\end{array}$ & 2007 & $\begin{array}{l}\text { Promoting the value } \\
\text { and practice of shared } \\
\text { decision-making in } \\
\text { mental healthcare }\end{array}$ & $\begin{array}{l}\text { Psychiatric Rehabilitation } \\
\text { Journal 3I(I): 54-6I }\end{array}$ & USA & Discussion & Discussion & Dyad & $\begin{array}{l}\text { PWLE- } \\
\text { Provider }\end{array}$ \\
\hline $\begin{array}{l}\text { Swanson, } \\
\text { K.A., } \\
\text { Bastani, R., } \\
\text { Rubenstein, } \\
\text { L.V. et al. }\end{array}$ & 2007 & $\begin{array}{l}\text { Effect of mental } \\
\text { healthcare and shared } \\
\text { decision-making on } \\
\text { patient satisfaction in a } \\
\text { community sample of } \\
\text { patients with depression }\end{array}$ & $\begin{array}{l}\text { Medical Care Research } \\
\text { and Review 64(4): } \\
416-30\end{array}$ & USA & Quantitative & $\begin{array}{l}\text { Cross- } \\
\text { sectional } \\
\text { survey }\end{array}$ & Dyad & $\begin{array}{l}\text { PWLE- } \\
\text { Provider }\end{array}$ \\
\hline $\begin{array}{l}\text { Buist, A., } \\
\text { Speelman, } \\
\text { C., Hayes, } \\
\text { B., Reay, R., } \\
\text { Milgrom, J. }\end{array}$ & 2007 & $\begin{array}{l}\text { Impact of education on } \\
\text { women with perinatal } \\
\text { depression }\end{array}$ & $\begin{array}{l}\text { Obstetrics \& Gynecology } \\
\text { 28(I): 49-54 }\end{array}$ & Australia & Quantitative & Pre-post & Dyad & $\begin{array}{l}\text { PWLE-- } \\
\text { Researcher }\end{array}$ \\
\hline
\end{tabular}


Knowledge Translation in Mental Health

\begin{tabular}{|c|c|c|c|c|c|c|c|c|}
\hline Authors & Year & Title & Publication & Country & $\begin{array}{l}\text { Research } \\
\text { approach }\end{array}$ & $\begin{array}{l}\text { Research } \\
\text { design }\end{array}$ & $\begin{array}{l}\text { Type of } \\
\text { exchange }\end{array}$ & Groups \\
\hline $\begin{array}{l}\text { Lucock, M., } \\
\text { Barber, R., } \\
\text { Jones, A., } \\
\text { Lovell, J. }\end{array}$ & 2007 & $\begin{array}{l}\text { Service users' views of } \\
\text { self-help strategies and } \\
\text { research in the UK }\end{array}$ & $\begin{array}{l}\text { Journal of Mental Health } \\
\text { 16(6): 795-805 }\end{array}$ & UK & Qualitative & $\begin{array}{l}\text { Participatory } \\
\text { action }\end{array}$ & Dyad & $\begin{array}{l}\text { PWLE- } \\
\text { Researcher }\end{array}$ \\
\hline Cole, N. & 2007 & $\begin{array}{l}\text { Depression awareness: } \\
\text { community-based } \\
\text { approach }\end{array}$ & $\begin{array}{l}\text { Australian Psychologist } \\
\text { 42(2): |6|-66 }\end{array}$ & Australia & Quantitative & $\begin{array}{l}\text { Pre-post } \\
\text { survey }\end{array}$ & Dyad & $\begin{array}{l}\text { Researcher- } \\
\text { Other }\end{array}$ \\
\hline $\begin{array}{l}\text { Hallett, J., } \\
\text { Brown, G., } \\
\text { Maycock, B., } \\
\text { Langdon, P. }\end{array}$ & 2007 & $\begin{array}{l}\text { Changing communities, } \\
\text { changing spaces: the } \\
\text { challenges of health } \\
\text { promotion outreach in } \\
\text { cyberspace }\end{array}$ & $\begin{array}{l}\text { Promotion \& Education } \\
\text { | 4(3): 150-54 }\end{array}$ & Australia & Qualitative & $\begin{array}{l}\text { Case study, } \\
\text { participatory } \\
\text { action } \\
\text { research }\end{array}$ & Dyad & $\begin{array}{l}\text { Researcher- } \\
\text { Other }\end{array}$ \\
\hline $\begin{array}{l}\text { Jacobson, N., } \\
\text { Ochocka, } \\
\text { J., Wise, J., } \\
\text { Janzen, R. }\end{array}$ & 2007 & $\begin{array}{l}\text { Inspiring knowledge } \\
\text { mobilization through } \\
\text { a communications } \\
\text { policy: the case of a } \\
\text { community-university } \\
\text { research alliance }\end{array}$ & $\begin{array}{l}\text { Progress in Community } \\
\text { Health Partnerships: } \\
\text { Research, Education and } \\
\text { Action I(I): 99-104 }\end{array}$ & Canada & Qualitative & $\begin{array}{l}\text { Participatory } \\
\text { action } \\
\text { research }\end{array}$ & Polyad & $\begin{array}{l}\text { Family- } \\
\text { Provider- } \\
\text { Policy maker- } \\
\text { Researcher }\end{array}$ \\
\hline Sax, P. & 2007 & $\begin{array}{l}\text { Finding common ground: } \\
\text { parents speak out about } \\
\text { family-centered practices }\end{array}$ & $\begin{array}{l}\text { Journal of Systemic } \\
\text { Therapies 26(3): 72-90 }\end{array}$ & USA & Qualitative & $\begin{array}{l}\text { Participatory } \\
\text { action }\end{array}$ & Polyad & $\begin{array}{l}\text { Family- } \\
\text { Provider- } \\
\text { Researcher }\end{array}$ \\
\hline $\begin{array}{l}\text { Garretsen, } \\
\text { H.F.L., } \\
\text { Bongers, } \\
\text { I.M.B., Roo, } \\
\text { A.A. de et al. }\end{array}$ & 2007 & $\begin{array}{l}\text { Bridging the gap between } \\
\text { science and practice: do } \\
\text { applied academic centres } \\
\text { contribute to a solution? }\end{array}$ & $\begin{array}{l}\text { Journal of Comparative } \\
\text { Social Welfare 23(I): } \\
\text { 49-59 }\end{array}$ & Netherlands & Discussion & $\begin{array}{l}\text { Discussion, } \\
\text { case examples }\end{array}$ & Polyad & $\begin{array}{l}\text { Provider- } \\
\text { Policy maker- } \\
\text { Researcher }\end{array}$ \\
\hline $\begin{array}{l}\text { Levesque, P., } \\
\text { Davidson, S., } \\
\text { Kidder, K. }\end{array}$ & 2007 & $\begin{array}{l}\text { Knowledge exchange } \\
\text { for attention deficit } \\
\text { hyperactivity disorder } \\
\text { research: an integrated } \\
\text { evidence and knowledge } \\
\text { exchange framework } \\
\text { leading to more effective } \\
\text { research dissemination } \\
\text { practices }\end{array}$ & $\begin{array}{l}\text { Journal of the Canadian } \\
\text { Academy of Child and } \\
\text { Adolescent Psychiatry } \\
\text { 16(2): 51-56 }\end{array}$ & Canada & Discussion & Discussion & Polyad & $\begin{array}{l}\text { Provider- } \\
\text { Policy maker- } \\
\text { Researcher- } \\
\text { Other }\end{array}$ \\
\hline $\begin{array}{l}\text { Nagel, T. \& } \\
\text { Thompson, C. }\end{array}$ & 2007 & $\begin{array}{l}\text { AIMHI NT "mental } \\
\text { health storyteller mob": } \\
\text { developing stories in } \\
\text { mental health }\end{array}$ & $\begin{array}{l}\text { Australian e-Journal for the } \\
\text { Advancement of Mental } \\
\text { Health 6(2): I-6 }\end{array}$ & Australia & Qualitative & $\begin{array}{l}\text { Participatory } \\
\text { action, survey }\end{array}$ & Polyad & $\begin{array}{l}\text { PWLE- } \\
\text { Family- } \\
\text { Provider- } \\
\text { Policy maker- } \\
\text { Researcher }\end{array}$ \\
\hline $\begin{array}{l}\text { Hoolihan, B., } \\
\text { Grosvenor, } \\
\text { J., Kurtz, H. } \\
\text { et al. }\end{array}$ & 2007 & $\begin{array}{l}\text { Utilizing technology } \\
\text { to raise mental health } \\
\text { literacy in small rural } \\
\text { towns }\end{array}$ & $\begin{array}{l}\text { Learning in Health and } \\
\text { Social Care 6(3): } 145-55\end{array}$ & Australia & Quantitative & $\begin{array}{l}\text { Cross- } \\
\text { sectional } \\
\text { survey }\end{array}$ & Polyad & $\begin{array}{l}\text { PWLE- } \\
\text { Provider- } \\
\text { Other }\end{array}$ \\
\hline
\end{tabular}


Elliot M. Goldner et al.

\begin{tabular}{|c|c|c|c|c|c|c|c|c|}
\hline Authors & Year & Title & Publication & Country & $\begin{array}{l}\text { Research } \\
\text { approach }\end{array}$ & $\begin{array}{l}\text { Research } \\
\text { design }\end{array}$ & $\begin{array}{l}\text { Type of } \\
\text { exchange }\end{array}$ & Groups \\
\hline $\begin{array}{l}\text { Moore, D.E., } \\
\text { Niebler, S.E., } \\
\text { Schlundt, D.G. } \\
\text { et al. }\end{array}$ & 2007 & $\begin{array}{l}\text { A conceptual model } \\
\text { for using action inquiry } \\
\text { technologies to address } \\
\text { disparities in depression }\end{array}$ & $\begin{array}{l}\text { Journal of Continuing } \\
\text { Education in the Health } \\
\text { Professions 27: 55-64 }\end{array}$ & USA & Discussion & $\begin{array}{l}\text { Discussion, } \\
\text { participatory } \\
\text { action } \\
\text { research, } \\
\text { conceptual } \\
\text { model }\end{array}$ & Polyad & $\begin{array}{l}\text { PWLE- } \\
\text { Provider- } \\
\text { Policy maker }\end{array}$ \\
\hline $\begin{array}{l}\text { Cleary, M., } \\
\text { Walter, G., } \\
\text { Luscombe, G. }\end{array}$ & 2007 & $\begin{array}{l}\text { Spreading the word: } \\
\text { disseminating research } \\
\text { results to patients and } \\
\text { carers }\end{array}$ & $\begin{array}{l}\text { Acta Neuropsychiatrica } \\
\text { 19(4): 224-29 }\end{array}$ & Australia & Discussion & Discussion & Polyad & $\begin{array}{l}\text { PWLE- } \\
\text { Provider- } \\
\text { Policy maker- } \\
\text { Researcher }\end{array}$ \\
\hline $\begin{array}{l}\text { Janzen, R., } \\
\text { Nelson, G., } \\
\text { Hausfather, } \\
\text { N., Ochocka, } \\
\text { J. }\end{array}$ & 2007 & $\begin{array}{l}\text { Capturing system-level } \\
\text { activities and impacts of } \\
\text { mental health consumer- } \\
\text { run organizations }\end{array}$ & $\begin{array}{l}\text { American Journal of } \\
\text { Community Psychology } \\
\text { 39(3/4): 287-99 }\end{array}$ & USA & Other & $\begin{array}{l}\text { Mixed } \\
\text { methods, } \\
\text { participatory } \\
\text { action } \\
\text { research }\end{array}$ & Polyad & $\begin{array}{l}\text { PWLE- } \\
\text { Provider- } \\
\text { Policy maker- } \\
\text { Researcher- } \\
\text { Other }\end{array}$ \\
\hline Spring, B. & 2007 & $\begin{array}{l}\text { Evidence-based practice } \\
\text { in clinical psychology: } \\
\text { what it is, why it matters } \\
\text { - what you need to } \\
\text { know }\end{array}$ & $\begin{array}{l}\text { Journal of Clinical } \\
\text { Psychology 63(7): 61 I-31 }\end{array}$ & USA & Discussion & Discussion & Polyad & $\begin{array}{l}\text { PWLE- } \\
\text { Provider- } \\
\text { Researcher }\end{array}$ \\
\hline Lind, $C$. & 2007 & $\begin{array}{l}\text { The power of adolescent } \\
\text { voices: co-researchers in } \\
\text { mental health promotion }\end{array}$ & $\begin{array}{l}\text { Educational Action } \\
\text { Research 15(3): 37|-83 }\end{array}$ & Canada & Qualitative & $\begin{array}{l}\text { Participatory } \\
\text { action }\end{array}$ & Polyad & $\begin{array}{l}\text { PWLE- } \\
\text { Provider- } \\
\text { Researcher- } \\
\text { Other }\end{array}$ \\
\hline $\begin{array}{l}\text { Bell, J.S., } \\
\text { Aslani, P., } \\
\text { McLachlan, } \\
\text { A.J., } \\
\text { Whitehead, P., } \\
\text { Chen, T. }\end{array}$ & 2007 & $\begin{array}{l}\text { Mental health case } \\
\text { conferences in primary } \\
\text { care: content and } \\
\text { treatment decision- } \\
\text { making }\end{array}$ & $\begin{array}{l}\text { Research in Social \& } \\
\text { Administrative Pharmacy } \\
\text { 3(1): 86-103 }\end{array}$ & Canada & Qualitative & $\begin{array}{l}\text { Case } \\
\text { conferences }\end{array}$ & Within group & $\begin{array}{l}\text { Provider- } \\
\text { Provider }\end{array}$ \\
\hline $\begin{array}{l}\text { Cohen, J. \& } \\
\text { Mannarino, } \\
\text { A.P. }\end{array}$ & 2008 & $\begin{array}{l}\text { Disseminating and } \\
\text { implementing trauma- } \\
\text { focused CBT in } \\
\text { community settings }\end{array}$ & $\begin{array}{l}\text { Trauma Violence Abuse } \\
9(4): 214-26\end{array}$ & USA & Other & Review & Dyad & $\begin{array}{l}\text { Provider- } \\
\text { Policy maker }\end{array}$ \\
\hline $\begin{array}{l}\text { Barwick, M.A., } \\
\text { Boydell, K.M., } \\
\text { Stasiulis, E. } \\
\text { et al. }\end{array}$ & 2008 & $\begin{array}{l}\text { Research utilization } \\
\text { among children's mental } \\
\text { health providers }\end{array}$ & $\begin{array}{l}\text { Implementation Science } \\
3(19)\end{array}$ & Canada & Quantitative & $\begin{array}{l}\text { Cross- } \\
\text { sectional } \\
\text { survey }\end{array}$ & Dyad & $\begin{array}{l}\text { Provider- } \\
\text { Researcher }\end{array}$ \\
\hline $\begin{array}{l}\text { Bilsker, D., } \\
\text { Anderson, J., } \\
\text { Samra, J. et al. }\end{array}$ & 2008 & $\begin{array}{l}\text { Behavioural interventions } \\
\text { in primary care: an } \\
\text { implementation trial }\end{array}$ & $\begin{array}{l}\text { Canadian Journal of } \\
\text { Community Mental Health } \\
\text { 27: 179-89 }\end{array}$ & Canada & Quantitative & Pre-post & Dyad & $\begin{array}{l}\text { Provider- } \\
\text { Researcher }\end{array}$ \\
\hline
\end{tabular}


Knowledge Translation in Mental Health

\begin{tabular}{|c|c|c|c|c|c|c|c|c|}
\hline Authors & Year & Title & Publication & Country & $\begin{array}{l}\text { Research } \\
\text { approach }\end{array}$ & $\begin{array}{l}\text { Research } \\
\text { design }\end{array}$ & $\begin{array}{l}\text { Type of } \\
\text { exchange }\end{array}$ & Groups \\
\hline $\begin{array}{l}\text { Boydell, KM., } \\
\text { Stasiulis, E., } \\
\text { Barwick, M. } \\
\text { et al. }\end{array}$ & 2008 & $\begin{array}{l}\text { Challenges of knowledge } \\
\text { translation in rural } \\
\text { communities: the case } \\
\text { of rural children's mental } \\
\text { health }\end{array}$ & $\begin{array}{l}\text { Canadian Journal of } \\
\text { Community Mental Health } \\
\text { 27(1): 49-63 }\end{array}$ & Canada & Qualitative & Focus groups & Dyad & $\begin{array}{l}\text { Provider- } \\
\text { Researcher }\end{array}$ \\
\hline $\begin{array}{l}\text { Chagnon, } \\
\text { F., Houle, J., } \\
\text { Daigle, M. } \\
\text { et al. }\end{array}$ & 2008 & $\begin{array}{l}\text { Application des } \\
\text { connaissances } \\
\text { scientifiques en } \\
\text { prévention du suicide : } \\
\text { vérification d'une } \\
\text { stratégie fondée sur la } \\
\text { communauté de pratique }\end{array}$ & Frontières 2I(I): 90-97 & Canada & Qualitative & $\begin{array}{l}\text { Community } \\
\text { of practice }\end{array}$ & Dyad & $\begin{array}{l}\text { Provider- } \\
\text { Researcher }\end{array}$ \\
\hline $\begin{array}{l}\text { Self, R., Rigby, } \\
\text { A., Leggett, } \\
\text { C., Paxton, R. }\end{array}$ & 2008 & $\begin{array}{l}\text { Clinical Decision Support } \\
\text { Tool: a rational needs- } \\
\text { based approach to } \\
\text { making clinical decisions }\end{array}$ & $\begin{array}{l}\text { Journal of Mental Health } \\
\text { 17(1): 33-48 }\end{array}$ & UK & Other & $\begin{array}{l}\text { Mixed } \\
\text { methods, } \\
\text { participatory } \\
\text { action, cluster } \\
\text { analysis, } \\
\text { concurrent } \\
\text { validity }\end{array}$ & Dyad & $\begin{array}{l}\text { Provider- } \\
\text { Researcher }\end{array}$ \\
\hline $\begin{array}{l}\text { Thompson, } \\
\text { A., Sullivan, } \\
\text { S.A., Barley, } \\
\text { M. et al. }\end{array}$ & 2008 & $\begin{array}{l}\text { The DEBIT trial: } \\
\text { an intervention to } \\
\text { reduce antipsychotic } \\
\text { polypharmacy prescribing } \\
\text { in adult psychiatry wards } \\
\text { - a cluster-randomised } \\
\text { controlled trial }\end{array}$ & $\begin{array}{l}\text { Psychological Medicine 38: } \\
705-15\end{array}$ & UK & Quantitative & $\begin{array}{l}\text { Pragmatic } \\
\text { cluster- } \\
\text { randomized } \\
\text { controlled } \\
\text { trial }\end{array}$ & Dyad & $\begin{array}{l}\text { Provider- } \\
\text { Researcher }\end{array}$ \\
\hline $\begin{array}{l}\text { Deegan, } \mathrm{P.}, \\
\text { Rapp, C., } \\
\text { Holter, } \mathrm{M} \text {. } \\
\text { et al. }\end{array}$ & 2008 & $\begin{array}{l}\text { A program to support } \\
\text { shared decision-making in } \\
\text { an outpatient psychiatric } \\
\text { medication clinic }\end{array}$ & $\begin{array}{l}\text { Psychiatric Services 59(6): } \\
603-05\end{array}$ & USA & Qualitative & Focus groups & Dyad & $\begin{array}{l}\text { PWLE- } \\
\text { Provider }\end{array}$ \\
\hline $\begin{array}{l}\text { Hamann, J., } \\
\text { Mendel, R.T., } \\
\text { Fink, B. et al. }\end{array}$ & 2008 & $\begin{array}{l}\text { Patients' and psychiatrists' } \\
\text { perceptions of clinical } \\
\text { decisions during } \\
\text { schizophrenia treatment }\end{array}$ & $\begin{array}{l}\text { Journal of Nervous and } \\
\text { Mental Disease 196(4): } \\
\text { 329-32 }\end{array}$ & Germany & Other & $\begin{array}{l}\text { Mixed } \\
\text { methods, } \\
\text { interviews and } \\
\text { questionnaires }\end{array}$ & Dyad & $\begin{array}{l}\text { PWLE- } \\
\text { Provider }\end{array}$ \\
\hline $\begin{array}{l}\text { Stringer, B., } \\
\text { Van Meijel, B., } \\
\text { De Vree, W. } \\
\text { et al. }\end{array}$ & 2008 & $\begin{array}{l}\text { User involvement in } \\
\text { mental healthcare: the } \\
\text { role of nurses }\end{array}$ & $\begin{array}{l}\text { Journal of Psychiatric and } \\
\text { Mental Health Nursing 15: } \\
678-83\end{array}$ & Netherlands & Other & $\begin{array}{l}\text { Literature } \\
\text { review }\end{array}$ & Dyad & $\begin{array}{l}\text { PWLE- } \\
\text { Provider }\end{array}$ \\
\hline $\begin{array}{l}\text { Moll, S. \& } \\
\text { Clements, E.P. }\end{array}$ & 2008 & $\begin{array}{l}\text { Workplace mental } \\
\text { health: developing an } \\
\text { employer resource } \\
\text { through partnerships in } \\
\text { knowledge translation }\end{array}$ & $\begin{array}{l}\text { Occupational Therapy } \\
\text { Now 10(5): 17-19 }\end{array}$ & Canada & Discussion & Discussion & Dyad & $\begin{array}{l}\text { Researcher- } \\
\text { Other }\end{array}$ \\
\hline $\begin{array}{l}\text { Han, S.S. \& } \\
\text { Weiss, B. }\end{array}$ & 2008 & $\begin{array}{l}\text { Sustainability of teacher } \\
\text { implementation of } \\
\text { school-based mental } \\
\text { health programs }\end{array}$ & $\begin{array}{l}\text { Journal of Abnormal Child } \\
\text { Psychology 33(6): 665-79 }\end{array}$ & USA & Other & Review & Polyad & $\begin{array}{l}\text { Provider- } \\
\text { Policy maker- } \\
\text { Other }\end{array}$ \\
\hline
\end{tabular}


Elliot M. Goldner et al.

\begin{tabular}{|c|c|c|c|c|c|c|c|c|}
\hline Authors & Year & Title & Publication & Country & $\begin{array}{l}\text { Research } \\
\text { approach }\end{array}$ & $\begin{array}{l}\text { Research } \\
\text { design }\end{array}$ & $\begin{array}{l}\text { Type of } \\
\text { exchange }\end{array}$ & Groups \\
\hline Grol, R. & 2008 & $\begin{array}{l}\text { Knowledge transfer in } \\
\text { mental healthcare: how } \\
\text { do we bring evidence } \\
\text { into day-to-day practice? }\end{array}$ & $\begin{array}{l}\text { Canadian Journal of } \\
\text { Psychiatry 53(5): 275-76 }\end{array}$ & Canada & Discussion & Discussion & Polyad & $\begin{array}{l}\text { Provider- } \\
\text { Policy maker- } \\
\text { Researcher }\end{array}$ \\
\hline Unutzer, J. & 2008 & $\begin{array}{l}\text { Evidence-based } \\
\text { treatments for anxiety } \\
\text { and depression: lost in } \\
\text { translation? }\end{array}$ & $\begin{array}{l}\text { Depression and Anxiety } \\
\text { 25(9): 726-29 }\end{array}$ & USA & Discussion & Discussion & Polyad & $\begin{array}{l}\text { Provider- } \\
\text { Policy maker- } \\
\text { Researcher }\end{array}$ \\
\hline $\begin{array}{l}\text { Smolders, M., } \\
\text { Laurant, M., } \\
\text { Roberge, P. } \\
\text { et al. }\end{array}$ & 2008 & $\begin{array}{l}\text { Knowledge transfer and } \\
\text { improvement of primary } \\
\text { and ambulatory care for } \\
\text { patients with anxiety }\end{array}$ & $\begin{array}{l}\text { Canadian Journal of } \\
\text { Psychiatry 53(5): 277-93 }\end{array}$ & Netherlands & Other & $\begin{array}{l}\text { Review with } \\
\text { meta-analysis }\end{array}$ & Polyad & $\begin{array}{l}\text { Provider- } \\
\text { Policy maker- } \\
\text { Researcher }\end{array}$ \\
\hline $\begin{array}{l}\text { Westhues, } \\
\text { A., Ochocka, } \\
\text { J., Jacobson, } \\
\text { N., Simich, } \\
\text { L., Maiter, } \\
\text { S., Janzen, R. } \\
\text { et al. }\end{array}$ & 2008 & $\begin{array}{l}\text { Developing theory from } \\
\text { complexity: reflections } \\
\text { on a collaborative mixed- } \\
\text { method participatory } \\
\text { action research study }\end{array}$ & $\begin{array}{l}\text { Qualitative Health } \\
\text { Research 18(5): 701-17 }\end{array}$ & Canada & Qualitative & $\begin{array}{l}\text { Participatory } \\
\text { action, } \\
\text { grounded } \\
\text { theory, } \\
\text { abduction, } \\
\text { synthesis }\end{array}$ & Polyad & $\begin{array}{l}\text { PWLE- } \\
\text { Family- } \\
\text { Provider- } \\
\text { Researcher- } \\
\text { Other }\end{array}$ \\
\hline $\begin{array}{l}\text { Lindamer, } \\
\text { L.A., } \\
\text { Lebowitz, } \\
\text { B.D., Hough, } \\
\text { R.L. }\end{array}$ & 2008 & $\begin{array}{l}\text { Public-academic } \\
\text { partnerships: improving } \\
\text { care for older persons } \\
\text { with schizophrenia } \\
\text { through an academic- } \\
\text { community partnership }\end{array}$ & $\begin{array}{l}\text { Psychiatric Services 59(3): } \\
236-39\end{array}$ & USA & Discussion & Case example & Polyad & $\begin{array}{l}\text { PWLE- } \\
\text { Provider- } \\
\text { Policy maker- } \\
\text { Researcher }\end{array}$ \\
\hline $\begin{array}{l}\text { Chambers, } \\
\text { D.A. }\end{array}$ & 2008 & $\begin{array}{l}\text { Advancing the science } \\
\text { of implementation: a } \\
\text { workshop summary }\end{array}$ & $\begin{array}{l}\text { Administration and Policy } \\
\text { in Mental Health \& Mental } \\
\text { Health Services Research } \\
35(1 / 2): 3-10\end{array}$ & USA & Discussion & Discussion & Polyad & $\begin{array}{l}\text { PWLE- } \\
\text { Provider- } \\
\text { Researcher }\end{array}$ \\
\hline $\begin{array}{l}\text { Patel, S.R., } \\
\text { Bakken, S., } \\
\text { Ruland, C. }\end{array}$ & 2008 & $\begin{array}{l}\text { Recent advances in } \\
\text { shared decision-making } \\
\text { for mental health }\end{array}$ & $\begin{array}{l}\text { Current Opinion in } \\
\text { Psychiatry 21(6): 606-12 }\end{array}$ & USA & Other & Review & Polyad & $\begin{array}{l}\text { PWLE- } \\
\text { Provider- } \\
\text { Researcher }\end{array}$ \\
\hline $\begin{array}{l}\text { Pickett- } \\
\text { Schenk, S.A., } \\
\text { Lippincott, } \\
\text { R.C., Bennett, } \\
\text { C. et al. }\end{array}$ & 2008 & $\begin{array}{l}\text { Improving knowledge } \\
\text { about mental illness } \\
\text { through family-led } \\
\text { education: the journey } \\
\text { of hope }\end{array}$ & $\begin{array}{l}\text { Psychiatric Services 59(I): } \\
49-56\end{array}$ & USA & Quantitative & $\begin{array}{l}\text { Randomized } \\
\text { trial with } \\
\text { waiting list } \\
\text { control group }\end{array}$ & Within group & Family-Family \\
\hline $\begin{array}{l}\text { Kimberly, J. \& } \\
\text { Cook, J.M. }\end{array}$ & 2008 & $\begin{array}{l}\text { Organizational } \\
\text { measurement and the } \\
\text { implementation of } \\
\text { innovation in mental } \\
\text { health services }\end{array}$ & $\begin{array}{l}\text { Administration and Policy } \\
\text { in Mental Health \& Mental } \\
\text { Health Research 35: } \\
\text { II-20 }\end{array}$ & USA & Other & Review & Within group & $\begin{array}{l}\text { Provider- } \\
\text { Provider }\end{array}$ \\
\hline
\end{tabular}


Knowledge Translation in Mental Health

\begin{tabular}{|c|c|c|c|c|c|c|c|c|}
\hline Authors & Year & Title & Publication & Country & $\begin{array}{l}\text { Research } \\
\text { approach }\end{array}$ & $\begin{array}{l}\text { Research } \\
\text { design }\end{array}$ & $\begin{array}{l}\text { Type of } \\
\text { exchange }\end{array}$ & Groups \\
\hline $\begin{array}{l}\text { Pignatiello, A., } \\
\text { Boydell, K., } \\
\text { Teshima, J., } \\
\text { Volpe, T. }\end{array}$ & 2008 & $\begin{array}{l}\text { Supporting primary } \\
\text { care through paediatric } \\
\text { telepsychiatry }\end{array}$ & $\begin{array}{l}\text { Canadian Journal of } \\
\text { Community Mental Health } \\
\text { 27(2): |39-5| }\end{array}$ & Canada & Qualitative & $\begin{array}{l}\text { Participant } \\
\text { evaluation }\end{array}$ & Within group & $\begin{array}{l}\text { Provider- } \\
\text { Provider }\end{array}$ \\
\hline $\begin{array}{l}\text { Franx, G., } \\
\text { Kroon, H., } \\
\text { Grimshaw, J. } \\
\text { et al. }\end{array}$ & 2008 & $\begin{array}{l}\text { Organizational change } \\
\text { to transfer knowledge } \\
\text { and improve quality } \\
\text { and outcomes of } \\
\text { care for patients with } \\
\text { severe mental illness: a } \\
\text { systematic overview of } \\
\text { reviews }\end{array}$ & $\begin{array}{l}\text { Canadian Journal of } \\
\text { Psychiatry 53(5): 294-305 }\end{array}$ & Netherlands & Other & $\begin{array}{l}\text { Systematic } \\
\text { review, } \\
\text { overview of } \\
\text { reviews }\end{array}$ & Within group & $\begin{array}{l}\text { Researcher- } \\
\text { Researcher }\end{array}$ \\
\hline $\begin{array}{l}\text { Pullmann, } \\
\text { M.D. }\end{array}$ & 2009 & $\begin{array}{l}\text { Participatory research } \\
\text { in systems of care for } \\
\text { children's mental health }\end{array}$ & $\begin{array}{l}\text { American Journal of } \\
\text { Community Psychology } \\
44(1 / 2): 43-53\end{array}$ & USA & Discussion & $\begin{array}{l}\text { Discussion, } \\
\text { participatory } \\
\text { action } \\
\text { framework }\end{array}$ & Dyad & $\begin{array}{l}\text { Family- } \\
\text { Researcher }\end{array}$ \\
\hline $\begin{array}{l}\text { Danseco, E., } \\
\text { Sundar, P., } \\
\text { Kasprzak, S. } \\
\text { et al. }\end{array}$ & 2009 & $\begin{array}{l}\text { Are we there yet? } \\
\text { Evaluation and the } \\
\text { knowledge translation } \\
\text { journey }\end{array}$ & $\begin{array}{l}\text { Journal of the Canadian } \\
\text { Academy of Child and } \\
\text { Adolescent Psychiatry } \\
\text { |8(I): 7-15 }\end{array}$ & Canada & Discussion & $\begin{array}{l}\text { Discussion, } \\
\text { case example }\end{array}$ & Dyad & $\begin{array}{l}\text { Policy maker- } \\
\text { Researcher }\end{array}$ \\
\hline $\begin{array}{l}\text { Isaac, M., } \\
\text { Elias, B., Katz, } \\
\text { L.Y., Belik, S., } \\
\text { Deane, F.P., } \\
\text { Enns, M.W., } \\
\text { Sareen, J. }\end{array}$ & 2009 & $\begin{array}{l}\text { Gatekeeper training as a } \\
\text { preventative intervention } \\
\text { for suicide: a systematic } \\
\text { review }\end{array}$ & $\begin{array}{l}\text { Canadian Journal of } \\
\text { Psychiatry } 54 \text { (4): 260-68 }\end{array}$ & Canada & Other & $\begin{array}{l}\text { Systematic } \\
\text { review }\end{array}$ & Dyad & $\begin{array}{l}\text { Provider- } \\
\text { Other }\end{array}$ \\
\hline $\begin{array}{l}\text { Stolee, P., } \\
\text { McAiney, } \\
\text { C.A., Hillier, } \\
\text { L.M. et al. }\end{array}$ & 2009 & $\begin{array}{l}\text { Sustained transfer of } \\
\text { knowledge to practice } \\
\text { in long-term care: } \\
\text { facilitators and barriers of } \\
\text { a mental health learning } \\
\text { initiative }\end{array}$ & $\begin{array}{l}\text { Gerontology \& Geriatrics } \\
\text { Education } 30(1): 1-20\end{array}$ & Canada & Qualitative & Interviews & Dyad & $\begin{array}{l}\text { Provider- } \\
\text { Policy maker }\end{array}$ \\
\hline $\begin{array}{l}\text { Barwick, M.A., } \\
\text { Peters, J., } \\
\text { Boydell, K.J. }\end{array}$ & 2009 & $\begin{array}{l}\text { Getting to uptake: } \\
\text { do communities of } \\
\text { practice support the } \\
\text { implementation of } \\
\text { evidence-based practice? }\end{array}$ & $\begin{array}{l}\text { Canadian Academy of } \\
\text { Child \& Adolescent } \\
\text { Psychiatry 18: 16-29 }\end{array}$ & Canada & Quantitative & $\begin{array}{l}\text { Randomized } \\
\text { controlled } \\
\text { trial }\end{array}$ & Dyad & $\begin{array}{l}\text { Provider- } \\
\text { Researcher }\end{array}$ \\
\hline $\begin{array}{l}\text { Brekke, J.S., } \\
\text { Phillips, E., } \\
\text { Pancake, L. } \\
\text { et al. }\end{array}$ & 2009 & $\begin{array}{l}\text { Implementation practice } \\
\text { and implementation } \\
\text { research: a report from } \\
\text { the field }\end{array}$ & $\begin{array}{l}\text { Research on Social Work } \\
\text { Practice I9S: 592-60 I }\end{array}$ & USA & Discussion & Discussion & Dyad & $\begin{array}{l}\text { Provider- } \\
\text { Researcher }\end{array}$ \\
\hline $\begin{array}{l}\text { Happell, B., } \\
\text { Moxham, L., } \\
\text { Reid-Searl, } \\
\text { K., Dwyer, } \\
\text { T., Kahl, J., } \\
\text { Morris, J. } \\
\text { et al. }\end{array}$ & 2009 & $\begin{array}{l}\text { Promoting mental } \\
\text { healthcare in a rural } \\
\text { paediatric unit through } \\
\text { participatory action } \\
\text { research }\end{array}$ & $\begin{array}{l}\text { Australian Journal of Rural } \\
\text { Health 17(3): 155-60 }\end{array}$ & Australia & Qualitative & Focus groups & Dyad & $\begin{array}{l}\text { Provider- } \\
\text { Researcher }\end{array}$ \\
\hline
\end{tabular}


Elliot M. Goldner et al.

\begin{tabular}{|c|c|c|c|c|c|c|c|c|}
\hline Authors & Year & Title & Publication & Country & $\begin{array}{l}\text { Research } \\
\text { approach }\end{array}$ & $\begin{array}{l}\text { Research } \\
\text { design }\end{array}$ & $\begin{array}{l}\text { Type of } \\
\text { exchange }\end{array}$ & Groups \\
\hline $\begin{array}{l}\text { Harpaz- } \\
\text { Rotem, I. \& } \\
\text { Rosenheck, } \\
\text { R.A. }\end{array}$ & 2009 & $\begin{array}{l}\text { Tracing the flow of } \\
\text { knowledge: geographic } \\
\text { variability in the } \\
\text { diffusion of prazosin } \\
\text { use for the treatment } \\
\text { of posttraumatic stress } \\
\text { disorder nationally in the } \\
\text { Department of Veterans } \\
\text { Affairs }\end{array}$ & $\begin{array}{l}\text { Archives of General } \\
\text { Psychiatry 66(4): 417-21 }\end{array}$ & USA & Quantitative & $\begin{array}{l}\text { Geographic } \\
\text { surveillance, } \\
\text { administrative } \\
\text { data analysis }\end{array}$ & Dyad & $\begin{array}{l}\text { Provider- } \\
\text { Researcher }\end{array}$ \\
\hline $\begin{array}{l}\text { Lamont, S., } \\
\text { Walker, P., } \\
\text { Brunero, S. }\end{array}$ & 2009 & $\begin{array}{l}\text { "Teaching an old dog } \\
\text { new tricks": a practice } \\
\text { development approach } \\
\text { to organizational change } \\
\text { in mental health }\end{array}$ & $\begin{array}{l}\text { Practice Development in } \\
\text { Health Care 8(2): 65-76 }\end{array}$ & Australia & Qualitative & $\begin{array}{l}\text { Participatory } \\
\text { action }\end{array}$ & Dyad & $\begin{array}{l}\text { Provider- } \\
\text { Researcher }\end{array}$ \\
\hline Nadeau, L. & 2009 & $\begin{array}{l}\text { Évaluation préliminaire } \\
\text { d'un projet de soins } \\
\text { concertés en santé } \\
\text { mentale jeunesse } \\
\text { à Montréal : faire } \\
\text { face à l'incertitude } \\
\text { institutionnelle et } \\
\text { culturelle }\end{array}$ & $\begin{array}{l}\text { Santé Mentale au Québec } \\
34(1): \text { 127-42 }\end{array}$ & Canada & Qualitative & Case study & Dyad & $\begin{array}{l}\text { Provider- } \\
\text { Researcher }\end{array}$ \\
\hline $\begin{array}{l}\text { Perreault, M., } \\
\text { Perreault, N., } \\
\text { Withaeuper, } \\
\text { D. et al. }\end{array}$ & 2009 & $\begin{array}{l}\text { Le Défi du traitement } \\
\text { et de la prévention des } \\
\text { troubles concomitants } \\
\text { sur la base de données } \\
\text { probantes }\end{array}$ & $\begin{array}{l}\text { Criminologie 42(1): } \\
91-114\end{array}$ & Canada & Discussion & Discussion & Dyad & $\begin{array}{l}\text { Provider- } \\
\text { Researcher }\end{array}$ \\
\hline $\begin{array}{l}\text { Robitaille, D. } \\
\text { \& Boudreault, } \\
\text { D. }\end{array}$ & 2009 & $\begin{array}{l}\text { Expérience d'une équipe } \\
\text { de santé mentale de } \\
\text { première ligne dans } \\
\text { l'optimisation des soins }\end{array}$ & $\begin{array}{l}\text { Santé Mentale au Québec } \\
34(1): 207-19\end{array}$ & Canada & Qualitative & Case study & Dyad & $\begin{array}{l}\text { Provider- } \\
\text { Researcher }\end{array}$ \\
\hline $\begin{array}{l}\text { Ward, V.L., } \\
\text { House, A.O., } \\
\text { Hamer, S. }\end{array}$ & 2009 & $\begin{array}{l}\text { Knowledge brokering: } \\
\text { exploring the process of } \\
\text { transferring knowledge } \\
\text { into action }\end{array}$ & $\begin{array}{l}\text { BMC Health Services } \\
\text { Research 16: 9-12 }\end{array}$ & UK & Other & $\begin{array}{l}\text { Description of } \\
\text { research plan }\end{array}$ & Dyad & $\begin{array}{l}\text { Provider- } \\
\text { Researcher }\end{array}$ \\
\hline Applbaum, K. & 2009 & $\begin{array}{l}\text { "Consumers are } \\
\text { patients!" Shared } \\
\text { decision-making and } \\
\text { treatment: non- } \\
\text { compliance as a business } \\
\text { opportunity }\end{array}$ & $\begin{array}{l}\text { Transcultural Psychiatry } \\
46(1): 107-30\end{array}$ & USA & Discussion & Discussion & Dyad & $\begin{array}{l}\text { PWLE- } \\
\text { Provider }\end{array}$ \\
\hline $\begin{array}{l}\text { Drake, R.E., } \\
\text { Cimpean, D., } \\
\text { Torrey, W.C. }\end{array}$ & 2009 & $\begin{array}{l}\text { Shared decision- } \\
\text { making in mental } \\
\text { health: prospects for } \\
\text { personalized medicine }\end{array}$ & $\begin{array}{l}\text { Dialogues in Clinical } \\
\text { Neuroscience I I(4): } \\
455-63\end{array}$ & USA & Discussion & Discussion & Dyad & $\begin{array}{l}\text { PWLE- } \\
\text { Provider }\end{array}$ \\
\hline
\end{tabular}


Knowledge Translation in Mental Health

\begin{tabular}{|c|c|c|c|c|c|c|c|c|}
\hline Authors & Year & Title & Publication & Country & $\begin{array}{l}\text { Research } \\
\text { approach }\end{array}$ & $\begin{array}{l}\text { Research } \\
\text { design }\end{array}$ & $\begin{array}{l}\text { Type of } \\
\text { exchange }\end{array}$ & Groups \\
\hline $\begin{array}{l}\text { Houle, J., } \\
\text { Lespérance, } \\
\text { F., Beaulieu, } \\
\text { M.D. }\end{array}$ & 2009 & $\begin{array}{l}\text { Partager la décision } \\
\text { dans le traitement de la } \\
\text { dépression }\end{array}$ & $\begin{array}{l}\text { Le Médecin du Québec } \\
44(10): 27-31\end{array}$ & Canada & Discussion & Discussion & Dyad & $\begin{array}{l}\text { PWLE- } \\
\text { Provider }\end{array}$ \\
\hline $\begin{array}{l}\text { Joosten, } \\
\text { E.A.G. et al. }\end{array}$ & 2009 & $\begin{array}{l}\text { Shared decision-making } \\
\text { reduces drug use and } \\
\text { psychiatric severity in } \\
\text { substance-dependent } \\
\text { patients }\end{array}$ & $\begin{array}{l}\text { Psychotherapy and } \\
\text { Psychosomatics 78(4): } \\
245-53\end{array}$ & Netherlands & Quantitative & $\begin{array}{l}\text { Randomized } \\
\text { controlled } \\
\text { trial }\end{array}$ & Dyad & $\begin{array}{l}\text { PWLE- } \\
\text { Provider }\end{array}$ \\
\hline $\begin{array}{l}\text { Vatne, S., } \\
\text { Bjornerem, } \\
\text { H., Hoem, E. }\end{array}$ & 2009 & $\begin{array}{l}\text { One approach to } \\
\text { improving the care for } \\
\text { depression and anxiety } \\
\text { disorders has been to } \\
\text { "package" evidence- } \\
\text { based efficacious } \\
\text { treatment components } \\
\text { into effective programs } \\
\text { of care }\end{array}$ & $\begin{array}{l}\text { Scandinavian Journal } \\
\text { of Caring Sciences 23: } \\
\text { 84-92 }\end{array}$ & Norway & Qualitative & Interviews & Dyad & $\begin{array}{l}\text { PWLE- } \\
\text { Provider }\end{array}$ \\
\hline McDaid, S. & 2009 & $\begin{array}{l}\text { An equality of condition } \\
\text { framework for user } \\
\text { involvement in mental } \\
\text { health policy and } \\
\text { planning: evidence from } \\
\text { participatory action } \\
\text { research }\end{array}$ & $\begin{array}{l}\text { Disability \& Society 24(4): } \\
461-74\end{array}$ & Ireland & Qualitative & $\begin{array}{l}\text { Participatory } \\
\text { action }\end{array}$ & Dyad & $\begin{array}{l}\text { PWLE- } \\
\text { Researcher }\end{array}$ \\
\hline $\begin{array}{l}\text { Oh, E., Jorm, } \\
\text { A.F., Wright, } \\
\text { A. }\end{array}$ & 2009 & $\begin{array}{l}\text { Perceived helpfulness } \\
\text { of websites for mental } \\
\text { health information }\end{array}$ & $\begin{array}{l}\text { Social Psychiatry \& } \\
\text { Psychiatric Epidemiology } \\
\text { 44(4): 293-99 }\end{array}$ & Australia & Quantitative & $\begin{array}{l}\text { Cross- } \\
\text { sectional } \\
\text { survey }\end{array}$ & Dyad & $\begin{array}{l}\text { Researcher- } \\
\text { Oher }\end{array}$ \\
\hline $\begin{array}{l}\text { Bapat, S., } \\
\text { Jorm, A., } \\
\text { Lawrence, K. }\end{array}$ & 2009 & $\begin{array}{l}\text { Evaluation of a mental } \\
\text { health literacy training } \\
\text { program for junior } \\
\text { sporting clubs }\end{array}$ & $\begin{array}{l}\text { Australasian Psychiatry 17: } \\
\text { 475-79 }\end{array}$ & Australia & Quantitative & Pre-post & Dyad & $\begin{array}{l}\text { Researcher- } \\
\text { Other }\end{array}$ \\
\hline $\begin{array}{l}\text { Blignault, I., } \\
\text { Woodland, } \\
\text { L., Ponzio, V. } \\
\text { et al. }\end{array}$ & 2009 & $\begin{array}{l}\text { Using a multifaceted } \\
\text { community intervention } \\
\text { to reduce stigma about } \\
\text { mental illness in an } \\
\text { Australian Macedonian } \\
\text { community }\end{array}$ & $\begin{array}{l}\text { Health Promotion Journal } \\
\text { of Australia 20(3): 227-33 }\end{array}$ & Australia & Quantitative & Pre-post & Dyad & $\begin{array}{l}\text { Researcher- } \\
\text { Other }\end{array}$ \\
\hline $\begin{array}{l}\text { López, Steven } \\
\text { R., Lara, M., } \\
\text { Kopelowicz, } \\
\text { A., Solano, S., } \\
\text { Foncerrada, } \\
\text { H. }\end{array}$ & 2009 & $\begin{array}{l}\text { La CLAve to increase } \\
\text { psychosis literacy } \\
\text { of Spanish-speaking } \\
\text { community residents and } \\
\text { family caregivers }\end{array}$ & $\begin{array}{l}\text { Journal of Consulting and } \\
\text { Clinical Psychology 77(4): } \\
763-74\end{array}$ & USA & Quantitative & Pre-post & Polyad & $\begin{array}{l}\text { Family- } \\
\text { Researcher- } \\
\text { Other }\end{array}$ \\
\hline
\end{tabular}


Elliot M. Goldner et al.

\begin{tabular}{|c|c|c|c|c|c|c|c|c|}
\hline Authors & Year & Title & Publication & Country & $\begin{array}{l}\text { Research } \\
\text { approach }\end{array}$ & $\begin{array}{l}\text { Research } \\
\text { design }\end{array}$ & $\begin{array}{l}\text { Type of } \\
\text { exchange }\end{array}$ & Groups \\
\hline $\begin{array}{l}\text { Chorpita, B.F. } \\
\text { \& Regan, J. }\end{array}$ & 2009 & $\begin{array}{l}\text { Dissemination of effective } \\
\text { mental health treatment } \\
\text { procedures: maximizing } \\
\text { the return on a significant } \\
\text { investment }\end{array}$ & $\begin{array}{l}\text { Behaviour Research and } \\
\text { Therapy 47(I I): 990-93 }\end{array}$ & USA & Discussion & Discussion & Polyad & $\begin{array}{l}\text { Provider- } \\
\text { Policy maker- } \\
\text { Researcher }\end{array}$ \\
\hline $\begin{array}{l}\text { Luck, J., } \\
\text { Hagigi, F., } \\
\text { Parker, L.E., } \\
\text { Yano, E.M., } \\
\text { Rubenstein, } \\
\text { L.V., Kirchner, } \\
\text { J.E. }\end{array}$ & 2009 & $\begin{array}{l}\text { A social marketing } \\
\text { approach to } \\
\text { implementing evidence- } \\
\text { based practice in VHA } \\
\text { QUERI: the TIDES } \\
\text { depression collaborative } \\
\text { care model }\end{array}$ & $\begin{array}{l}\text { Implementation Science } \\
4: 64\end{array}$ & USA & Qualitative & Case study & Polyad & $\begin{array}{l}\text { Provider- } \\
\text { Policy maker- } \\
\text { Researcher }\end{array}$ \\
\hline $\begin{array}{l}\text { Perreault, M., } \\
\text { Wiethaueper, } \\
\text { D., Perreault, } \\
\text { N., Bonin, } \\
\text { J.-P., Brown, } \\
\text { T.G., Brunaud, } \\
\text { H. }\end{array}$ & 2009 & $\begin{array}{l}\text { Meilleures pratiques } \\
\text { et formation dans le } \\
\text { contexte du continuum } \\
\text { des services en } \\
\text { santé mentale et } \\
\text { en toxicomanie : le } \\
\text { programme de formation } \\
\text { croisée du sud-ouest de } \\
\text { Montréal }\end{array}$ & $\begin{array}{l}\text { Santé Mentale au Québec } \\
34(1): 143-60\end{array}$ & Canada & Qualitative & Case study & Polyad & $\begin{array}{l}\text { Provider- } \\
\text { Researcher- } \\
\text { Other }\end{array}$ \\
\hline $\begin{array}{l}\text { Curran, J. \& } \\
\text { Newton, M. }\end{array}$ & 2009 & $\begin{array}{l}\text { Theme issue on } \\
\text { knowledge translation }\end{array}$ & $\begin{array}{l}\text { Journal of the Canadian } \\
\text { Academy of Child and } \\
\text { Adolescent Psychiatry } \\
\text { |8(1): 2-3 }\end{array}$ & Canada & Discussion & $\begin{array}{l}\text { Discussion, } \\
\text { editorial }\end{array}$ & Polyad & $\begin{array}{l}\text { PWLE- } \\
\text { Family- } \\
\text { Provider- } \\
\text { Policy maker- } \\
\text { Researcher }\end{array}$ \\
\hline $\begin{array}{l}\text { Harrison, A., } \\
\text { \& Brandling, J. }\end{array}$ & 2009 & $\begin{array}{l}\text { Improving mental } \\
\text { healthcare for older } \\
\text { people within a general } \\
\text { hospital in the UK }\end{array}$ & $\begin{array}{l}\text { Nursing \& Health Sciences } \\
\text { I I (3): 293-300 }\end{array}$ & UK & Qualitative & $\begin{array}{l}\text { Participatory } \\
\text { action } \\
\text { research }\end{array}$ & Polyad & $\begin{array}{l}\text { PWLE- } \\
\text { Family- } \\
\text { Provider- } \\
\text { Researcher }\end{array}$ \\
\hline $\begin{array}{l}\text { Manion, l., } \\
\text { Buchanan, } \\
\text { D.H., Cheng, } \\
\text { M. et al. }\end{array}$ & 2009 & $\begin{array}{l}\text { Embedding evidence- } \\
\text { based practice in child } \\
\text { and youth mental health } \\
\text { in Ontario }\end{array}$ & $\begin{array}{l}\text { Evidence \& Policy: A } \\
\text { Journal of Research, } \\
\text { Debate \& Practice 5(2): } \\
|4|-53\end{array}$ & Canada & Discussion & $\begin{array}{l}\text { Discussion, } \\
\text { case examples }\end{array}$ & Polyad & $\begin{array}{l}\text { PWLE- } \\
\text { Family- } \\
\text { Provider- } \\
\text { Researcher- } \\
\text { Other }\end{array}$ \\
\hline $\begin{array}{l}\text { de Wolff, A., } \\
\text { Cabezas, P., } \\
\text { Chamberlain, } \\
\text { L. et al. }\end{array}$ & 2009 & $\begin{array}{l}\text { The creation of "We } \\
\text { Are Neighbours": } \\
\text { participatory research } \\
\text { and recovery }\end{array}$ & $\begin{array}{l}\text { Canadian Journal of } \\
\text { Community Mental Health } \\
28(2): 61-72\end{array}$ & Canada & Qualitative & $\begin{array}{l}\text { Participatory } \\
\text { action } \\
\text { research }\end{array}$ & Polyad & $\begin{array}{l}\text { PWLE-Policy } \\
\text { maker- } \\
\text { Researcher }\end{array}$ \\
\hline $\begin{array}{l}\text { Mitton, C., } \\
\text { Adair, C.E., } \\
\text { McKenzie, } \\
\text { E., Patten, S., } \\
\text { Waye-Perry, } \\
\text { B., Smith, N. }\end{array}$ & 2009 & $\begin{array}{l}\text { Designing a knowledge } \\
\text { transfer and exchange } \\
\text { strategy for the Alberta } \\
\text { Depression Initiative: } \\
\text { contributions of } \\
\text { qualitative research with } \\
\text { key stakeholders }\end{array}$ & $\begin{array}{l}\text { International Journal of } \\
\text { Mental Health Systems } \\
3(1): 11\end{array}$ & Canada & Qualitative & Focus groups & Polyad & $\begin{array}{l}\text { PWLE- } \\
\text { Provider- } \\
\text { Policy maker }\end{array}$ \\
\hline
\end{tabular}


Knowledge Translation in Mental Health

\begin{tabular}{|c|c|c|c|c|c|c|c|c|}
\hline Authors & Year & Title & Publication & Country & $\begin{array}{l}\text { Research } \\
\text { approach }\end{array}$ & $\begin{array}{l}\text { Research } \\
\text { design }\end{array}$ & $\begin{array}{l}\text { Type of } \\
\text { exchange }\end{array}$ & Groups \\
\hline $\begin{array}{l}\text { McGrath, P.J., } \\
\text { Lingley-Pottie, } \\
\text { E., Thurston, } \\
\text { C., McLean, } \\
\text { C. }\end{array}$ & 2009 & $\begin{array}{l}\text { Integrated knowledge } \\
\text { translation in mental } \\
\text { health: family help as an } \\
\text { example }\end{array}$ & $\begin{array}{l}\text { Journal of the Canadian } \\
\text { Academy of Child and } \\
\text { Adolescent Psychiatry 18: } \\
\text { 30-37 }\end{array}$ & Canada & Qualitative & Case study & Polyad & $\begin{array}{l}\text { PWLE- } \\
\text { Provider- } \\
\text { Policy maker- } \\
\text { Researcher }\end{array}$ \\
\hline $\begin{array}{l}\text { Drake, R.E., } \\
\text { Wilkniss, S.M., } \\
\text { Frounfelker, } \\
\text { R.L., et al. }\end{array}$ & 2009 & $\begin{array}{l}\text { The Thresholds- } \\
\text { Dartmouth partnership } \\
\text { and research on shared } \\
\text { decision-making }\end{array}$ & $\begin{array}{l}\text { Psychiatric Services 60(2): } \\
\text { |42-44 }\end{array}$ & USA & Discussion & Discussion & Polyad & $\begin{array}{l}\text { PWLE- } \\
\text { Provider- } \\
\text { Researcher }\end{array}$ \\
\hline Lyons, J.S. & 2009 & $\begin{array}{l}\text { Knowledge creation } \\
\text { through total clinical } \\
\text { outcomes management: } \\
\text { a practice-based evidence } \\
\text { solution to address some } \\
\text { of the challenges of } \\
\text { knowledge translation }\end{array}$ & $\begin{array}{l}\text { Journal of the Canadian } \\
\text { Academy of Child and } \\
\text { Adolescent Psychiatry } \\
\text { |8(I): 38-45 }\end{array}$ & Canada & Discussion & $\begin{array}{l}\text { Discussion, } \\
\text { case sample } \\
\text { of KE } \\
\text { framework }\end{array}$ & Polyad & $\begin{array}{l}\text { PWLE- } \\
\text { Provider- } \\
\text { Researcher }\end{array}$ \\
\hline $\begin{array}{l}\text { Maar, M.A., } \\
\text { Erskine, B., } \\
\text { McGregor, L., } \\
\text { Larose, T.L., } \\
\text { Sutherland, } \\
\text { M.E., Graham, } \\
\text { D. et al. }\end{array}$ & 2009 & $\begin{array}{l}\text { Innovations on a } \\
\text { shoestring: a study of a } \\
\text { collaborative community- } \\
\text { based Aboriginal mental } \\
\text { health service model in } \\
\text { rural Canada }\end{array}$ & $\begin{array}{l}\text { International Journal of } \\
\text { Mental Health Systems } \\
3: 27\end{array}$ & Canada & Qualitative & $\begin{array}{l}\text { Participatory } \\
\text { action, } \\
\text { ethnographic }\end{array}$ & Polyad & $\begin{array}{l}\text { PWLE- } \\
\text { Provider- } \\
\text { Researcher }\end{array}$ \\
\hline $\begin{array}{l}\text { Gozlan, G. } \\
\text { Acef, S., } \\
\text { Petitqueux- } \\
\text { Glaser, C. }\end{array}$ & 2009 & $\begin{array}{l}\text { Vers des soins intégrés } \\
\text { en santé mentale: } \\
\text { l'expérience du réseau } \\
\text { Prépsy }\end{array}$ & $\begin{array}{l}\text { Santé Mentale au Québec } \\
\text { 34(I): 221-38 }\end{array}$ & France & Discussion & Case example & Within group & $\begin{array}{l}\text { Provider- } \\
\text { Provider }\end{array}$ \\
\hline $\begin{array}{l}\text { Hamilton, } \\
\text { A.B., Cohen, } \\
\text { A.N., Young, } \\
\text { A.S. }\end{array}$ & 2009 & $\begin{array}{l}\text { Organizational readiness } \\
\text { in specialty mental } \\
\text { healthcare }\end{array}$ & $\begin{array}{l}\text { Journal of General Internal } \\
\text { Medicine } 25 \text { (Suppl. I): } \\
\text { 27-31 }\end{array}$ & USA & Other & $\begin{array}{l}\text { Mixed } \\
\text { methods, } \\
\text { questionnaire } \\
\text { and semi- } \\
\text { structured } \\
\text { interviews }\end{array}$ & Within group & $\begin{array}{l}\text { Provider- } \\
\text { Provider }\end{array}$ \\
\hline $\begin{array}{l}\text { Proctor, E.K., } \\
\text { Landsverk, J., } \\
\text { Aarons, G. } \\
\text { et al. }\end{array}$ & 2009 & $\begin{array}{l}\text { Implementation research } \\
\text { in mental health } \\
\text { services: an emerging } \\
\text { science with conceptual, } \\
\text { methodological, and } \\
\text { training challenges }\end{array}$ & $\begin{array}{l}\text { Administration and Policy } \\
\text { in Mental Health \& Mental } \\
\text { Health Services Research } \\
36(1): 24-34\end{array}$ & USA & Discussion & Discussion & Within group & $\begin{array}{l}\text { Researcher- } \\
\text { Researcher }\end{array}$ \\
\hline $\begin{array}{l}\text { Lévesque, L., } \\
\text { Ducharme, } \\
\text { F., Caron, C. } \\
\text { et al. }\end{array}$ & 2010 & $\begin{array}{l}\text { A partnership approach } \\
\text { to service needs } \\
\text { assessment with family } \\
\text { caregivers of an aging } \\
\text { relative living at home: a } \\
\text { qualitative analysis of the } \\
\text { experiences of caregivers } \\
\text { and practitioners }\end{array}$ & $\begin{array}{l}\text { International Journal of } \\
\text { Nursing Studies 47(7): } \\
876-87\end{array}$ & Canada & Qualitative & $\begin{array}{l}\text { Thematic } \\
\text { analysis }\end{array}$ & Dyad & $\begin{array}{l}\text { Family- } \\
\text { Provider }\end{array}$ \\
\hline
\end{tabular}


Elliot M. Goldner et al.

\begin{tabular}{|c|c|c|c|c|c|c|c|c|}
\hline Authors & Year & Title & Publication & Country & $\begin{array}{l}\text { Research } \\
\text { approach }\end{array}$ & $\begin{array}{l}\text { Research } \\
\text { design }\end{array}$ & $\begin{array}{l}\text { Type of } \\
\text { exchange }\end{array}$ & Groups \\
\hline $\begin{array}{l}\text { Byrne, M.K., } \\
\text { Willis, A., } \\
\text { Deane, F.P. } \\
\text { et al. }\end{array}$ & 2010 & $\begin{array}{l}\text { Training inpatient } \\
\text { mental health staff how } \\
\text { to enhance patient } \\
\text { engagement with } \\
\text { medications: Medication } \\
\text { Alliance training and } \\
\text { dissemination outcomes } \\
\text { in a large US mental } \\
\text { health hospital }\end{array}$ & $\begin{array}{l}\text { Journal of Evaluation in } \\
\text { Clinical Practice 16(1): } \\
\text { 114-20 }\end{array}$ & USA & Quantitative & Pre-post & Dyad & $\begin{array}{l}\text { Provider- } \\
\text { Researcher }\end{array}$ \\
\hline $\begin{array}{l}\text { Moxham, L., } \\
\text { Dwyer, T., } \\
\text { Happell, B., } \\
\text { Reid-Searl, } \\
\text { K., Kahl, J., } \\
\text { Morris, J., } \\
\text { et al. }\end{array}$ & 2010 & $\begin{array}{l}\text { Recognising our role: } \\
\text { improved confidence of } \\
\text { general nurses providing } \\
\text { care to young people } \\
\text { with a mental illness in a } \\
\text { rural paediatric unit }\end{array}$ & $\begin{array}{l}\text { Journal of Clinical Nursing } \\
\text { 19(9/10): 1434-42 }\end{array}$ & Australia & Qualitative & $\begin{array}{l}\text { Participatory } \\
\text { action }\end{array}$ & Dyad & $\begin{array}{l}\text { Provider- } \\
\text { Researcher }\end{array}$ \\
\hline $\begin{array}{l}\text { Gray, R., } \\
\text { White, J., } \\
\text { Schulz, M. } \\
\text { et al. }\end{array}$ & 2010 & $\begin{array}{l}\text { Enhancing medication } \\
\text { adherence in people } \\
\text { with schizophrenia: an } \\
\text { international programme } \\
\text { of research }\end{array}$ & $\begin{array}{l}\text { International Journal of } \\
\text { Mental Health Nursing } \\
\text { 19(I): 36-44 }\end{array}$ & UK & Other & $\begin{array}{l}\text { Mixed } \\
\text { methods, } \\
\text { concept } \\
\text { mapping }\end{array}$ & Dyad & $\begin{array}{l}\text { PWLE- } \\
\text { Provider }\end{array}$ \\
\hline $\begin{array}{l}\text { Glisson, C., } \\
\text { Schoenwald, } \\
\text { S.K., } \\
\text { Hemmelgarn, } \\
\text { A. et al. }\end{array}$ & 2010 & $\begin{array}{l}\text { Randomized trial of } \\
\text { MST and ARC in a } \\
\text { two-level evidence- } \\
\text { based treatment } \\
\text { implementation strategy }\end{array}$ & $\begin{array}{l}\text { Journal of Consulting and } \\
\text { Clinical Psychology 78(4): } \\
537-50\end{array}$ & USA & Quantitative & $\begin{array}{l}\text { Randomized } \\
\text { controlled } \\
\text { trial }\end{array}$ & Polyad & $\begin{array}{l}\text { Family- } \\
\text { Provider- } \\
\text { Policy maker- } \\
\text { Other }\end{array}$ \\
\hline $\begin{array}{l}\text { Duncan, E., } \\
\text { Best, C., } \\
\text { Hagen, S. }\end{array}$ & 2010 & $\begin{array}{l}\text { Shared decision-making } \\
\text { interventions for people } \\
\text { with mental health } \\
\text { conditions }\end{array}$ & $\begin{array}{l}\text { Cochrane Database } \\
\text { of Systematic Reviews } \\
\text { (January) } 20(1)\end{array}$ & UK & Other & $\begin{array}{l}\text { Systematic } \\
\text { review }\end{array}$ & Polyad & $\begin{array}{l}\text { PWLE- } \\
\text { Provider- } \\
\text { Other }\end{array}$ \\
\hline Lane et al. & 2010 & $\begin{array}{l}\text { Des guides novateurs } \\
\text { pour soutenir } \\
\text { l'amélioration des } \\
\text { pratiques en CSSS }\end{array}$ & $\begin{array}{l}\text { Psychologie Québec } \\
\text { 27(I): 31-33 }\end{array}$ & Canada & Discussion & Discussion & Polyad & $\begin{array}{l}\text { PWLE- } \\
\text { Provider- } \\
\text { Policy maker- } \\
\text { Researcher }\end{array}$ \\
\hline
\end{tabular}

